Draft Version June 3, 2022

Preprint typeset using $\mathrm{LATE}_{\mathrm{E}} \mathrm{X}$ style emulateapj v. 03/07/07

\title{
ABELL 1201: THE ANATOMY OF A COLD FRONT CLUSTER FROM COMBINED OPTICAL AND X-RAY DATA
}

\author{
Matt S. Owers $^{1}$, Paul E.J. Nulsen ${ }^{2}$, Warrick J. Couch ${ }^{3}$, Maxim Markevitch ${ }^{2}$ and Gregory B. Poole ${ }^{3}$ \\ Draft version June 3, 2022
}

\begin{abstract}
We present a combined X-ray and optical analysis of the cold front cluster Abell 1201 using archival Chandra data and multi-object spectroscopy taken with the $3.9 \mathrm{~m}$ Anglo Australian and 6.5m Multiple Mirror Telescopes. This paper represents the first in a series presenting a study of a sample of cold front clusters selected from the Chandra archives with the aim of relating cold fronts to merger activity, understanding the dynamics of mergers and their effect on the cluster constituents. The Chandra X-ray imagery of Abell 1201 reveals two conspicuous surface brightness discontinuities, which are shown to be cold fronts, and a remnant core structure. Temperature maps reveal a complex multiphase temperature structure with regions of hot gas interspersed with fingers of cold gas. Our optical analysis is based on a sample of 321 confirmed members, whose mean redshift is $z=0.1673 \pm 0.0002$ and velocity dispersion is $778 \pm 36 \mathrm{~km} \mathrm{~s}^{-1}$. We search for dynamical substructure and find clear evidence for multiple localized velocity substructures coincident with over-densities in the galaxy surface density. Most notably, we find structure coincident with the remnant X-ray core. Despite the clear evidence for dynamical activity, we find the peculiar velocity distribution does not deviate significantly from Gaussian. We apply two-body dynamical analyses in order to assess which of the substructures are bound, and thus dynamically important in terms of the cluster merger history. We propose that the cold fronts in Abell 1201 are a consequence of its merger with a smaller subunit, which has induced gas motions that gave rise to 'sloshing' cold fronts. Abell 1201 illustrates the value of combining multi-wavelength data and multiple substructure detection techniques when attempting to ascertain the dynamical state of a cluster.
\end{abstract}

Subject headings: galaxies: clusters: individual (Abell 1201) - X-rays: galaxies: clusters

\section{INTRODUCTION}

Within the current cosmological paradigm, large scale structure in the Universe is expected to form in a hierarchical manner. This "bottom up" formation scenario culminates with the formation of clusters of galaxies, which are the largest and most massive virialized objects in the Universe. At the current epoch, a high fraction of clusters are still growing through the infall of matter, much of which is funneled through the surrounding spider-web like filamentary structures. The most extreme growth event occurs when two clusters of roughly equal mass merge, the merging process being one of the most energetic in the Universe, releasing around $10^{64}$ ergs of gravitational binding energy. Some $10 \%$ of this is dissipated via mechanisms such as shock and adiabatic heating of the intracluster medium (ICM), acceleration of relativistic particles, and generation of peculiar velocities and turbulence in the ICM (for a review of these physical processes see Sarazin 2002).

In this context, the new generation of X-ray observatories (XMM-Newton and Chandra) have provided a wealth of new information on the ICM and hence new insights into this cluster merger phenomenon. One of the first interesting discoveries - attributed to the excellent spatial resolution and sensitivity of Chandrawas the observation of extended 'edge' features in the

\footnotetext{
${ }^{1}$ School of Physics, University of New South Wales, Sydney, NSW 2052, Australia; mowers@phys.unsw.edu.au

${ }^{2}$ Harvard Smithsonian Center for Astrophysics, 60 Garden Street, Cambridge, MA 02138, USA

${ }^{3}$ Center for Astrophysics and Supercomputing, Swinburne University of Technology, Hawthorn, VIC 3122, Australia
}

X-ray surface brightness maps obtained for Abell 2142 (Markevitch et al. 2000) and Abell 3667 (Vikhlinin et al. 2001). The sharp discontinuity in surface brightness seen in Abell 3667 had been observed previously with ROSAT, and was interpreted as a shock front (Markevitch et al. 1999). However, the Chandra observations showed that in both Abell 2142 and Abell 3667, the temperature of the gas on the brighter (denser) side of the discontinuity was colder than the temperature of the less dense downstream gas - opposite to expectations for a shock. Furthermore, the temperature and density profiles across the discontinuities showed, in combination, that the pressure is roughly continuous, again inconsistent with a shock front. Thus, the observed edges appear to be contact discontinuities between cool, dense, low entropy gas and hotter, diffuse ambient ICM, leading them to be dubbed "cold fronts". Initial explanations attributed the low entropy gas to the remnant cooling core of a merging subcluster (Markevitch et al. 2000).

As the number of clusters observed to have cold front features has increased, it has become clear that the remnant core scenario is not the correct interpretation in all cases, since a number of cold fronts are observed to exist in clusters with an otherwise relaxed X-ray morphology (Mazzotta et al. 2001a,b; Markevitch et al. 2001; Markevitch \& Vikhlinin 2007). One such example is Abell 1795, the observations of which led Markevitch et al. (2001) to propose an alternative scenario whereby the 'sloshing' of the cooling core within the stationary gravitational potential well causes cool central gas to be displaced and a cold front formed where it comes into contact with higher entropy gas at larger 
radii.

Hydrodynamic simulations have an integral role in aiding the interpretation of these cluster X-ray observations and further understanding the underlying physics. Both Ascasibar \& Markevitch (2006) and Poole et al. (2006) found a plethora of transient cold-front like phenomena associated with sub-cluster gas arising during their simulated mergers, including the classic ram-pressure stripped remnant sub-cluster core preceded by a merger shock. Tittley \& Henriksen (2005) explored the possibility of forming a cold front through gas sloshing, finding it is possible to produce similar edges to those observed through core oscillations, although in their model they find the gravitational potential well oscillates due to the motion of the dark matter, inducing motion in the gas. Churazov et al. (2003) and Fujita et al. (2004) also showed that gas oscillations can be induced when the cluster core is displaced from the potential well by a weak shock or acoustic wave which has passed through the cluster center. Ascasibar \& Markevitch (2006) found that oscillation of the central gas and dark matter can easily be induced by infalling sub-clusters, and can even be induced due to the infall of a dark matter only subcluster. The gas core decouples from the dark matter when a rapid change in the direction of oscillation due to the core passage of the dark matter sub-cluster causes a change in the ram pressure felt by the gas, displacing it from the dark matter. The gas sloshing then occurs when the displaced gas falls back toward the potential minimum, generating edges at the turn-around point of each oscillation.

Whatever the mechanism, it appears the existence of a cold front can generally be interpreted as strong evidence of a system which is in the process of, or has recently undergone a merger. What has been lacking to date is a systematic study of the relationship between cold fronts and other dynamical indicators of cluster merger activity using multi-wavelength observations, which can be incorporated into detailed models in order to garner a complete understanding of how a major merger impacts the different cluster mass components. To address this issue, we have conducted a search of the Chandra archives and selected a sample of clusters exhibiting robust examples of cold fronts (the selection criteria and sample will be presented in a forthcoming paper; Owers et al. 2008, in prep.) for optical multiple-object spectra (MOS) and radio follow up observations. This paper represents the first of a series where we aim to show a relationship between cold fronts and merger activity through detection of substructure at optical wavelengths. Subsequent papers will relate the star formation/radio properties of the galaxies and large scale diffuse radio halo/relic emission associated with cluster mergers (Giovannini \& Feretti 2002).

Detection of substructure within clusters using optical data has a long history. Initially, galaxy surface density contours were used to search for projected galaxy concentrations within clusters (Geller \& Beers 1982). Advances in multi-object spectroscopy have allowed simultaneous observations of tens, and now hundreds of galaxy spectra within a field, meaning large samples of spectroscopically confirmed cluster members can now be compiled. Radial velocity information aids in eliminating the projection effects inherent in galaxy surface density contour methods, and a number of methods have been proposed which use the combination of galaxy radial velocity and spatial information to develop a statistic for the robust detection of substructures within a cluster (eg. see Dressler \& Shectman 1988; West \& Bothun 1990; Colless \& Dunn 1996; Girardi \& Biviano 2002). Since different statistics are sensitive to different types of substructure (Pinkney et al. 1996), the use of a combination of a number of different statistical methods is essential in diagnosing the dynamical activity in a cluster. Combining these substructure detection methods with data at X-ray wavelengths has proven to be a powerful tool in diagnosing cluster merger scenarios and is essential for disentangling the complex histories of merging systems (eg. Boschin et al. 2004; Girardi et al. 2006; Barrena et al. 2007; Carrasco et al. 2007; Maurogordato et al. 2008).

Here, in this first paper, we present a detailed optical and X-ray analysis of the cluster Abell 1201. The (previously unpublished) Chandra observations of this cluster reveal two sharp surface brightness discontinuities, both of which are cold fronts, and also an offset core of X-ray emission. Based on comparisons of the simulations of Poole et al. (2006) and Ascasibar \& Markevitch (2006) and these observations, Abell 1201 appears to be an excellent example of cold fronts generated by core gas motions caused by a gravitational perturbation in the form of a merging subcluster. Thus, Abell 1201 provides a unique opportunity to test this scenario using combined optical and X-ray analyses.

Abell 1201 is a richness class 2 cluster (Abell et al. 1989) at moderate redshift $\mathrm{z}=0.168$ (Struble \& Rood 1999) with an X-ray luminosity $\mathrm{L}_{\mathrm{x}}=2.4 \times 10^{44} \mathrm{ergs} \mathrm{s}^{-1}$ (Böhringer et al. 2000). The analysis presented is based on comprehensive optical spectroscopy obtained using both AAOmega on the Anglo-Australian Telescope (AAT) and Hectospec on the Multiple-Mirror Telescope (MMT) combined with Chandra archival X-ray data. The structure of the paper is as follows: In \$2 we present the reduction and analysis of the Chandra data. In $\$ 3$ we present the new MOS optical observations and analysis. In 4 we present a scenario for the merger history and formation of the cold fronts in Abell 1201. In $\$ 5$ we summarize our results.

Throughout the paper, we assume a standard $\Lambda \mathrm{CDM}$ cosmology where $H_{0}=70 \mathrm{~km} \mathrm{~s}^{-1}, \Omega_{m}=0.3$ and $\Omega_{\Lambda}=$ 0.7 . For this cosmology and at the redshift of the cluster $1^{\prime \prime}=2.88 \mathrm{kpc}$.

\section{X-RAY OBSERVATIONS AND ANALYSIS}

Our X-ray analysis of Abell 1201 uses Chandra archival data that were taken on 2004 November 3 (ObsId 4216). The observations were made with the ACIS-S array with the cluster centered on the back illuminated S3 chip, for a total exposure time of $40 \mathrm{ksec}$.

\subsection{Data preparation}

The data were reprocessed using the CIAO software package (version 3.4) starting from the standard Chandra pipeline processed level-1 event list. Observation specific bad pixel files containing hot pixel and cosmic ray afterglow information were produced and applied, the latest gain files and calibrations were applied and VFAINT mode cleaning was used for improved rejection of cosmic ray events. The data were then filtered to include only 
events with $A S C A$ grades 0, 2, 3, 4 and 6 .

Since the backside illuminated chips are prone to flare contamination, we filtered the data for periods of anomalously high background. We followed standard procedure and extracted lightcurves from source-free regions to search for flares. Cluster emission covers much of the S3 chip, so we extracted a lightcurve in the 2.5$6 \mathrm{keV}$ range using the S1 chip which has very similar flare properties to the S3 chip. The observation suffered from a strong flare, and approximately half the exposure time was affected, leaving a useful exposure time of $21.5 \mathrm{ks}$. During the spectral analyses presented below, this cleaned data is used. For the imaging analyses, including the fitting of surface brightness profiles, the full $40 \mathrm{ks}$ uncleaned exposure is used.

Background data were taken from the blank sky observations appropriate for the epoch of observation ${ }^{4}$. The backgrounds are taken from observations with low Galactic foregrounds and soft X-ray brightness, so we check the soft X-ray flux in the vicinity of Abell 1201 using the ROSAT all sky $\mathrm{R} 45^{5}$ count rates and confirm they are consistent with the blank sky background rates. The background and source data were processed using the same calibration files, bad pixel files and background filtering, with the backgrounds being reprojected to match the observations.

We check for residual backgrounds in the cleaned data by extracting spectra from the S1 chip and after subtracting backgrounds from the blank sky observations, find a small residual background. This residual was modeled by a soft thermal MEKAL component with solar abundance and $\mathrm{kT}=0.27 \mathrm{keV}$ (similar to that found in Markevitch et al. 2003) plus a cutoff power law, which is not folded through the instrument response, with photon index of -0.15 and exponential cutoff at $5.6 \mathrm{keV}$ (Markevitch et al. 2003). Including these components, scaled for region size, in the spectral fits performed in $\S 2.3$ makes no significant difference to the results.

\subsection{X-ray images and Surface Brightness Modeling}

The raw $0.5-7.0 \mathrm{keV}$ Chandra image, uncorrected for exposure and showing the entire ACIS-S3 chip, is presented in the left panel of Figure 1. The right panel of Figure 1 shows the corresponding exposure corrected, adaptively smoothed Chandra image. The cluster has a bright core, and appears elongated along an axis pointing to the north-west. Along this axis, there are three distinct features apart from the cool core: There are two surface brightness discontinuities, one to the south-east $\sim 300 \mathrm{kpc}$ from the core running from position angle (PA; measured from due west) $240^{\circ}$ to $267^{\circ}$ and one $\sim 50 \mathrm{kpc}$ north-west of the core $\left(\mathrm{PA} 8^{\circ}\right.$ to $120^{\circ}$ ) and $\sim 430 \mathrm{kpc}$ to the north-west there is a faint, diffuse excess clump of emission. We discuss the discontinuities further in $\$ 2.3 .2$ In Figure 2 we show the SDSS r-band optical image of the central regions of Abell 1201 with Chandra X-ray contours overlaid. The X-ray peak is offset by $\sim 2.8^{\prime \prime}$ from the brightest cluster galaxy (BCG) and the excess clump of emission approximately coincides with a second concentration of galaxies, which will be discussed further in $\$ 3$ and $\$ 4$.

\footnotetext{
${ }^{4}$ See http://cxc.harvard.edu/contrib/maxim/acisbg/

${ }^{5}$ See http://heasarc.gsfc.nasa.gov/docs/tools.html
}

\subsubsection{Beta model}

In order to emphasize the structures seen in Figure 1 we use the method of Neumann \& Bohringer (1997). The surface brightness distribution is fitted with an azimuthally symmetric model and the residuals are inspected for significant deviations. We use the Sherpa fitting package to fit a background subtracted doubleBeta model of the form

$$
S(r)=\sum_{i=1}^{2} S_{0, i}\left[1+\left(\frac{r}{r_{0, i}}\right)^{2}\right]^{-3 \beta+1 / 2}+B,
$$

where $r$ is the radius, measured from an adjustable origin. One Beta model accounts for the excess emission within the central $100 \mathrm{kpc}$ and the second models the remaining cluster emission. For fitting, the model is multiplied by the exposure map to account for instrumental effects. The best fitting model parameters are presented in Table 1

We obtain residual maps by subtracting the smooth model described above from the entire cluster image. This residual map is smoothed with a Gaussian kernel with $\sigma=5.9^{\prime \prime}$ and residual significance is determined using the method of Neumann \& Bohringer (1997), where Gaussian smoothing and Poissonian statistics are used to determine an error map for the X-ray image, and the significance map is the ratio of the residual to error map. Figure 3 shows the residual significance map, with the north-west clump detected at greater than $10 \sigma$ significance while an excess plume is detected in the direction of the south-east discontinuity. There appears to be a significant excess detected in the south-west at the bottom of the chip. This can be attributed to excess emission due to the background flare, since the excess is much less significant when the same analysis is performed on the cleaned $21.5 \mathrm{ks}$ exposure. Also apparent are the significant negative residuals surrounding the south-east plume and in the outer parts of the chip to the west, artifacts of the azimuthally symmetric model.

\subsection{Global spectral properties and the nature of the surface brightness discontinuities}

In this section we derive global properties for Abell 1201, and also analyze the properties of the sharp surface brightness discontinuities seen in Figure 1 with the aim of determining the nature of the fronts. Table 2 summarizes the various best fitting spectral parameters measured in the following sections.

\subsubsection{Global Temperature}

We derived a global temperature and metallicity for Abell 1201 within an elliptical region covering the majority of the cluster emission (see Figure 4). The spectrum was extracted using the CIAO dmextract tool, and point sources detected with wavdetect excised. The CIAO tool mkwarf was used to create the auxiliary response file $(\mathrm{ARF})$, which accounts for spatial variations in quantum efficiency (QE), effective area and the buildup of contaminant on the filter windows. The observation was performed at $-120^{\circ} \mathrm{C}$, so the CIAO tool mkacisrmf was used to create redistribution matrix files (RMF). The ARF and RMF calculated within a region are weighted by the number of events in the $0.5-2 \mathrm{keV}$ range within the region. 

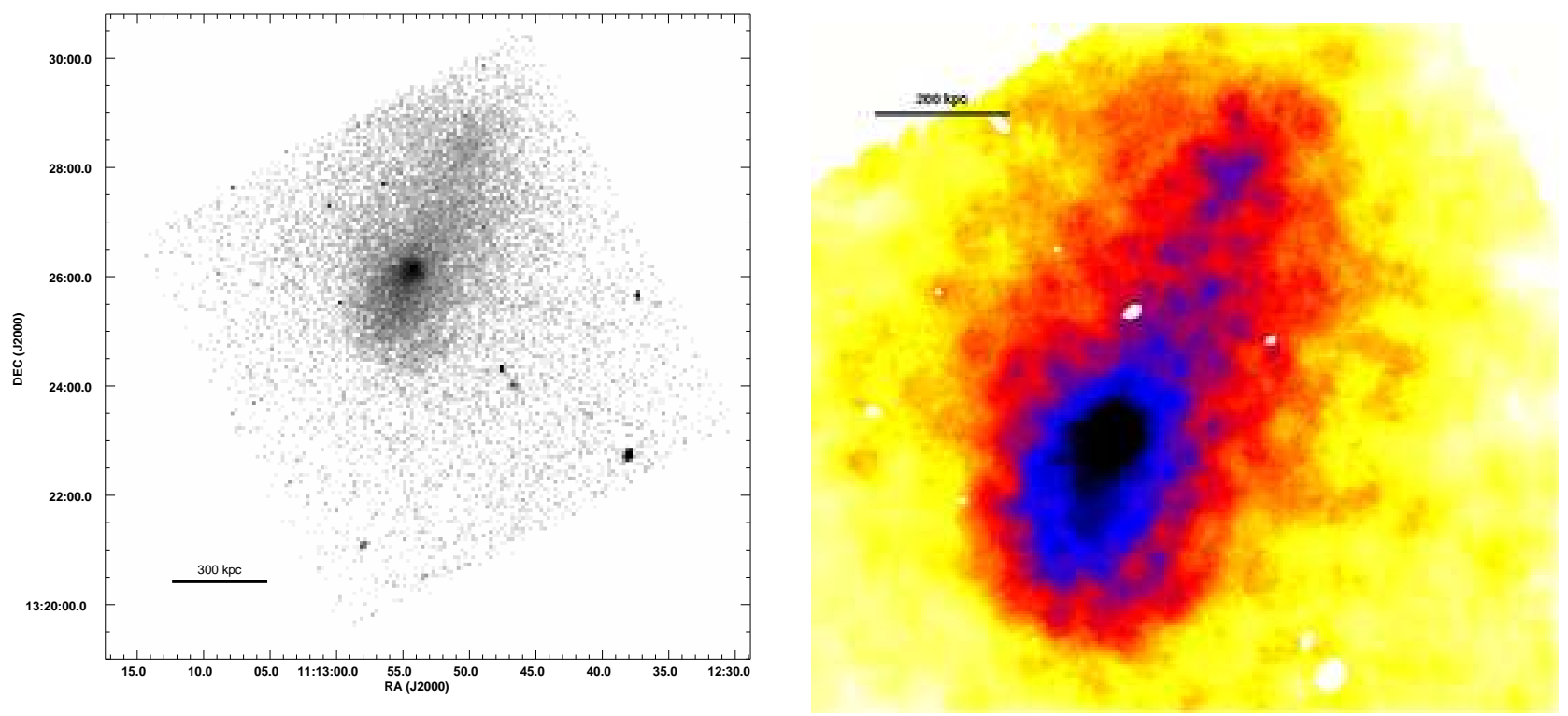

FIG. 1.- Left: Raw Chandra ACIS-S3 X-ray image of Abell 1201 in the 0.5-7.0 keV energy range, binned to $4^{\prime \prime}$ pixels, including only the S3 chip, using the full 40ks exposure and displayed on a logarithmic scale to enhance the diffuse, low surface brightness emission. North is up and east is to the left. Right: Adaptively smoothed, exposure corrected close up version of the left image with point sources removed. The emission appears elongated on an axis from the south-east to north-west, and there is a bright central X-ray core. To the north-west there is a clump of excess emission and there are two surface brightness discontinuities, one to the south-east and one near the core.

TABLE 1

BETA MODEL PARAMETERS DERIVED FROM FITTING THE SURFACE BRIGHTNESS DISTRIBUTION With A DOUble BETA MODEL (SEE TEXT).

\begin{tabular}{ccccccc}
\hline \hline $\begin{array}{c}x_{0}, y_{0} \\
(\mathrm{deg}, \mathrm{J} 2000)\end{array}$ & $\begin{array}{c}S_{0,1} \\
\left(10^{-7}\right)\end{array}$ & $\begin{array}{c}S_{0,2} \\
\left(10^{-7}\right)\end{array}$ & $\beta$ & $\begin{array}{c}r_{0,1} \\
(\mathrm{kpc})\end{array}$ & $\begin{array}{c}r_{0,2} \\
(\mathrm{kpc})\end{array}$ & $\begin{array}{c}B \\
\left(10^{-9}\right)\end{array}$ \\
\hline $11^{\mathrm{h}} 12^{\mathrm{m}} 54.3^{\mathrm{s}},+13^{\circ} 26^{\prime} 06.7^{\prime \prime}$ & $3.53_{-0.32}^{+0.38}$ & $0.44_{-0.04}^{+0.03}$ & $0.50_{-0.04}^{+0.04}$ & $22_{-3}^{+4}$ & $213_{-24}^{+22}$ & $6.56_{-0.54}^{+0.67}$
\end{tabular}

Note. - Units of $S_{0,1}, S_{0,2}$ and $B$ are photons $/ \mathrm{cm}^{2} / \mathrm{s} / \operatorname{arcsec}^{2}$. Errors on $x_{0}$ and $y_{0}$ are $\sim 0.4^{\prime \prime}$.

TABLE 2

Summary of BeSt FitTing SPECTRAL PARAmeters FOR SEVERAL REGIONS.

\begin{tabular}{ccc}
\hline \hline Region & $\begin{array}{c}\mathrm{kT} \\
(\mathrm{keV})\end{array}$ & $\begin{array}{c}\text { Abundance } \\
(Z)\end{array}$ \\
\hline Global & $5.3 \pm 0.3$ & $0.34 \pm 0.10$ \\
Inside south-east front & $3.6_{-0.7}^{+1.0}$ & $\ldots$ \\
Outside south-east front & $5.7_{-1.7}^{+3.7}$ & $\ldots$ \\
Inside north-west front & $3.2_{-0.7}^{+1.0}$ & $\ldots$ \\
Outside north-west front & $5.2_{-1.0}^{+1.5}$ & $\ldots$ \\
\hline
\end{tabular}

The unbinned spectra were fitted in the $0.5-9.8 \mathrm{keV}$ range using the MEKAL Kaastra 1992; Liedahl et al. 1995) and WABS models within the XSPEC package (Arnaud 1996), minimizing the Cash statistic. The MEKAL component models a hot, diffuse, single temperature plasma, and is multiplied by WABS, a photoelectric absorption model accounting for Galactic absorption, where the neutral hydrogen column density is fixed to the Galactic value of $N_{H}=1.61 \times 10^{20}$ (Dickev \& Lockman 1990). The best fitting values for the temperatures and abundance are $k T=5.3_{-0.3}^{+0.3}$ and $Z=0.34_{-0.10}^{+0.10}$ where the errors are $90 \%$ confidence limits. We used the LUMIN function to determine the integrated unabsorbed X-ray luminosity from the best fitting model in the energy range $0.5-7.0 \mathrm{keV}$, and obtained $L_{\mathrm{X}}(0.5-7.0 \mathrm{keV})=3.7 \times 10^{44} \mathrm{erg} \mathrm{s}^{-1}$ within the $r \simeq 550 \mathrm{kpc}$ elliptical aperture shown in Figure 4

\subsubsection{Surface Brightness Discontinuities}

To characterize the surface brightness discontinuities as either shock or cold fronts, we fitted the surface brightness profiles across them with a density model, and also measured the temperature difference across the discontinuities. The density model consists of a broken power law function such that

$$
n_{e}(r)= \begin{cases}n_{e, 1}\left(\frac{r}{R_{f}}\right)^{-\alpha_{1}}, & r<R_{f}, \\ n_{e, 2}\left(\frac{r}{R_{f}}\right)^{-\alpha_{2}}, & r>R_{f},\end{cases}
$$

where $R_{f}$ is the radius at which the discontinuity occurs, $n_{e, 1}$ and $n_{e, 2}$ are the densities of the inner and outer gas at $R_{f}$, respectively, and the spheroidal radius, $r$, is defined by $r^{2}=\varpi^{2}+\epsilon_{\zeta}^{2} \zeta^{2}$. Here $\zeta$ is the coordinate along our line of sight and the elliptical radius, $\varpi$, is defined by

$$
\varpi^{2}=\frac{\left.\epsilon^{2}[x \cos \theta+y \sin \theta]^{2}+[y \cos \theta-x \sin \theta)\right]^{2}}{\epsilon^{2}},
$$

where $x$ and $y$ are cartesian coordinates centered at the center of curvature of the discontinuity and $\theta$ determines 


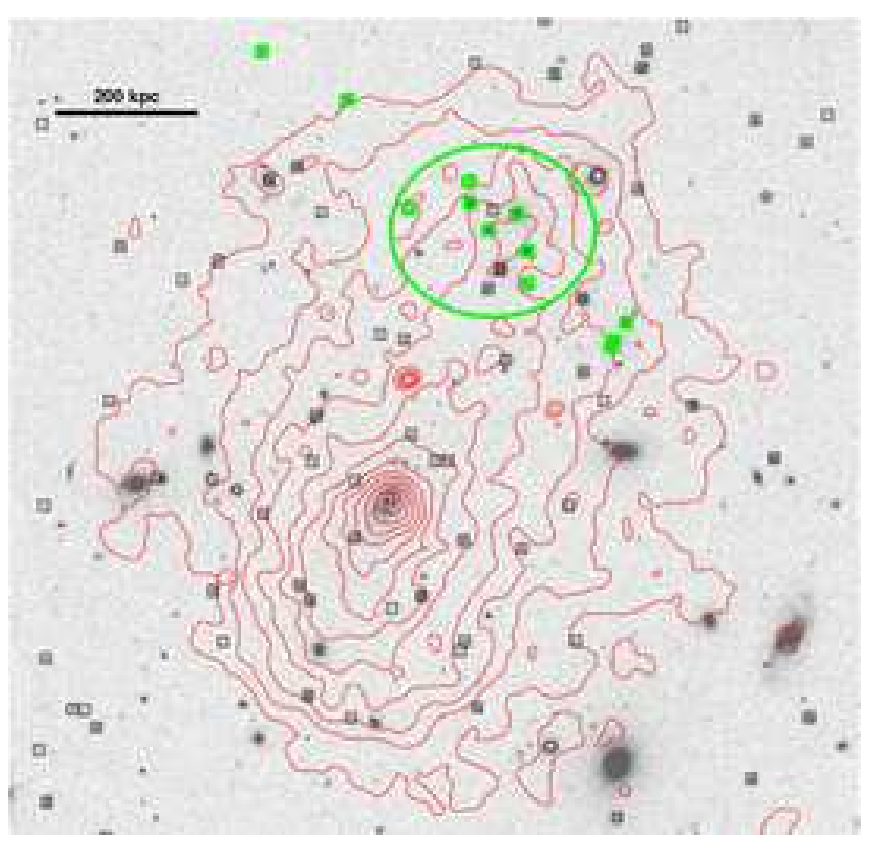

FIG. 2.- SDSS r-band image of the central region of Abell 1201. The red contours are the Chandra X-ray contours logarithmically spaced by a factor of 1.3 in the interval $1.5 \times 10^{-8}-4.6 \times 10^{-7}$ photons $\mathrm{cm}^{-2} \mathrm{~s}^{-1}$ pixel $^{-1}$, the black squares identify galaxies which are cluster members, the green squares show galaxies allocated to KMM1, and the green ellipse shows the $1 \sigma$ region for KMM1 from the KMM analysis (see $\S 3.3 .3$. The bright cluster galaxy white square appears to be associated with the bright X-ray core, although is offset by $\sim 2.8^{\prime \prime}$.

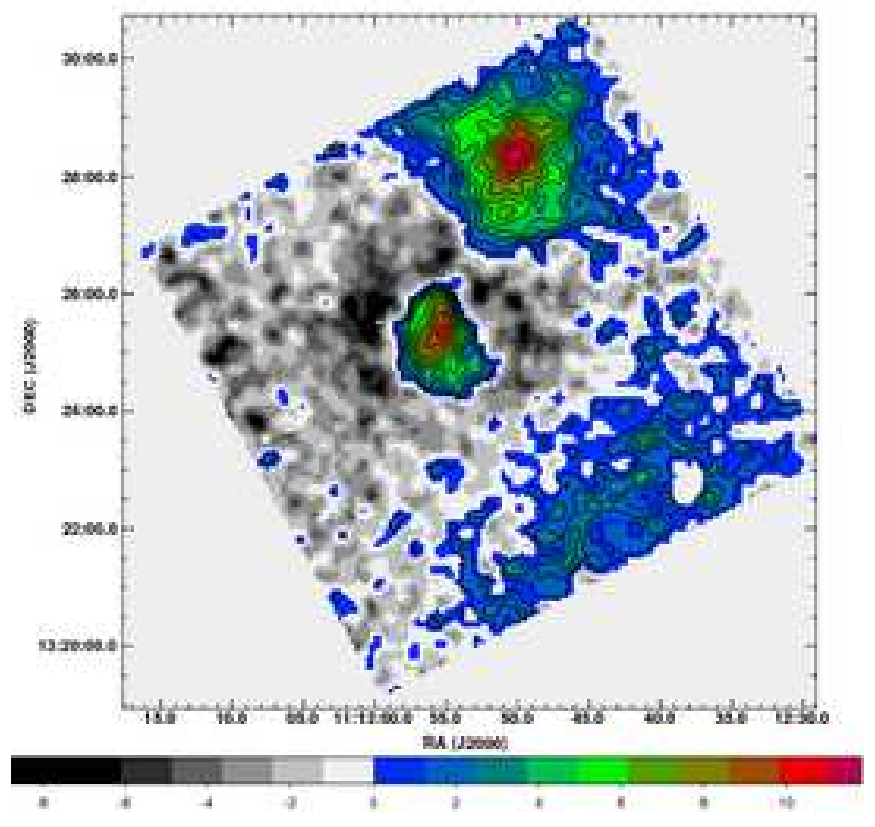

FIG. 3.- Residual significance map derived from subtracting a smooth double Beta model from the X-ray image and calculating the significance (see text). Grey scales show the negative residuals, whilst color scales show the positive residuals. The black contours range from $1-12 \sigma$ with linear increments of $1 \sigma$.

the orientation of the front on the plane of the sky. We assumed the gas density distributions inside and outside the front have the same ellipticities and have rotational symmetry so that $\epsilon_{\zeta}=1 / \epsilon$. The model is incorporated into Sherpa where we fitted the surface brightness across

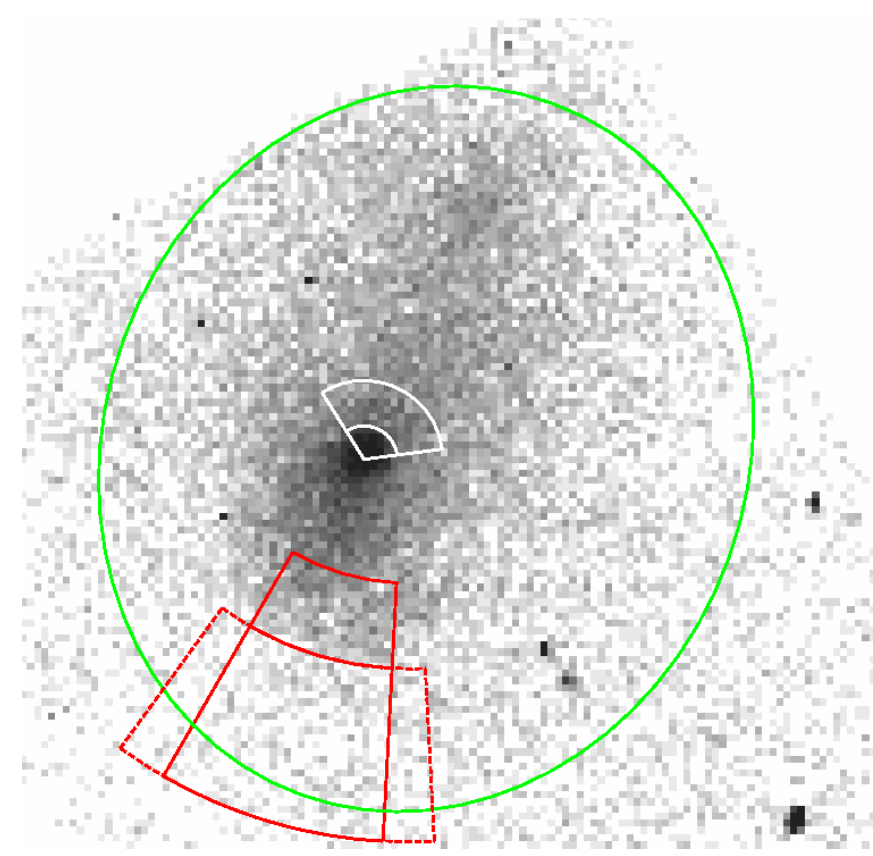

FIG. 4.- Close up of the raw Chandra image with regions showing where surface brightness profiles were fitted, and where spectra were extracted for temperature measurements. The large green ellipse shows the region where spectra were extracted for global temperature and abundance measurements. The surface brightness profiles shown in Figure 5 are taken from the solid red and white sectors. The boundaries indicate the fronts, and delineate regions where the temperature inside and outside the fronts are measured. The dashed red sector indicates the slightly larger opening angle used in measuring the temperature outside the south-east front.

the discontinuity in the sectors shown in Figure 4 using the full $40 \mathrm{ks}$ exposure, in the energy range $0.5-7 \mathrm{keV}$, and also add to the model a constant background component which was measured in $\S 2.2 .1$. We present the surface brightness distribution, along with the best fitting density models, in Figure 5. From the amplitudes of the best fitting surface brightness model, $A_{1}$ and $A_{2}$, we derived a density jump of $\sqrt{A_{1} / A_{2}}=n_{e, 1} / n_{e, 2}=2.13_{-0.39}^{+0.56}$ for the south-east discontinuity and $n_{e, 1} / n_{e, 2}=1.75_{-0.34}^{+0.47}$ for the north-west discontinuity. The confidence range for the density jump was computed from the extremes of the $90 \%$ confidence ranges for $A_{1}$ and $A_{2}$, assuming a single parameter of interest. We note that if the density jump is the only parameter of interest, this overestimates its confidence range.

The temperature profile across the front is an essential diagnostic tool. Ideally, to minimize projection effects we would like to measure the deprojected temperature profile across the fronts, however the limited number of photons available do not allow this. Instead, we extracted spectra from two regions for each front, one on the bright side of the front (inside) and one on the faint side (outside). The regions from which we extracted spectra for both the south-east and north-west fronts are shown in Figure 4. For the north-west front, we extracted spectra from one sector centered at $\mathrm{RA}=168.2268, \mathrm{DEC}=13.4344$ with PA from $7^{\circ}-122^{\circ}$ and radii $0-18.4^{\prime \prime}$ and one with the same center and PA, but with radii $18.4-45.5^{\prime \prime}$, corresponding to the sector in which we fit the density model above. For the south-east front, we extracted spectra in one sector centered at $\mathrm{RA}=168.2197, \mathrm{DEC}=13.45094$ with PA from $240^{\circ}-267^{\circ}$ and radii $127.9-175.1^{\prime \prime}$, and 

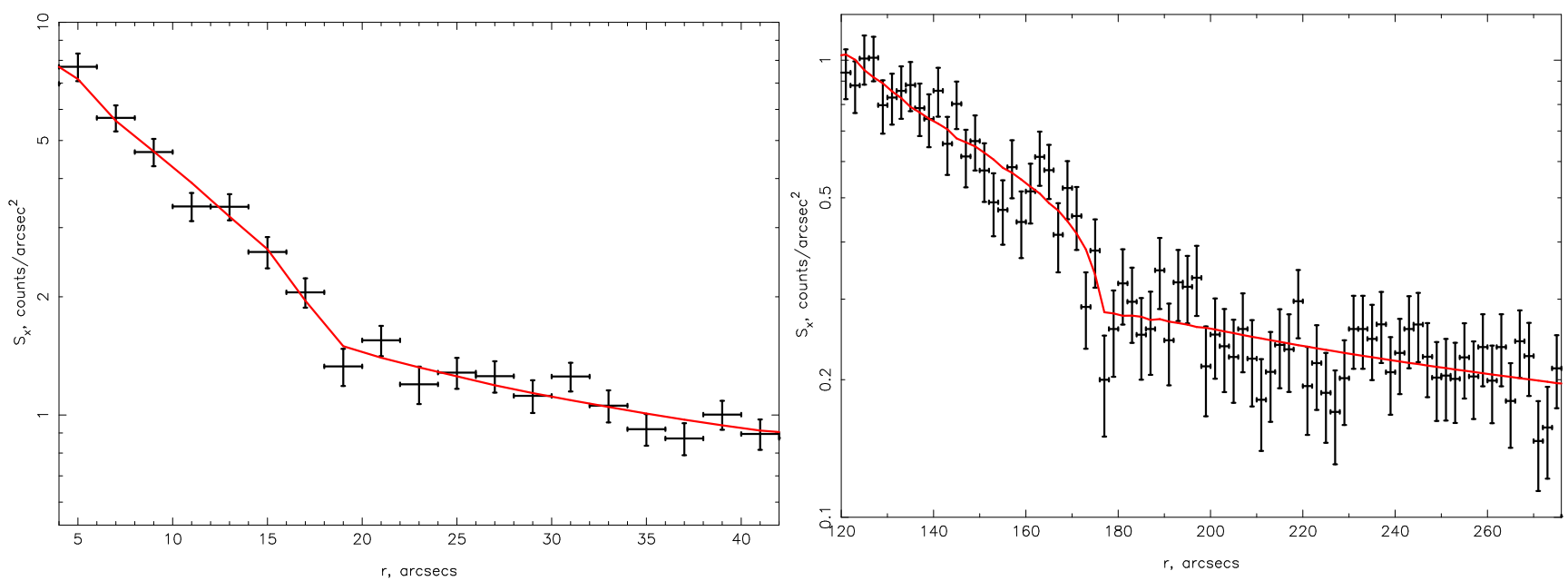

Fig. 5. - Left: Surface brightness profile across the north-west discontinuity measured in a sector from PA $7^{\circ}-122^{\circ}$ centered at the center of curvature of the front $(\mathrm{RA}=168.2268, \mathrm{DEC}=13.4344)$. Right: Surface brightness profile across the south-east discontinuity measured in a sector from PA $240^{\circ}-267^{\circ}$ centered at the center of curvature of the front $(\mathrm{RA}=168.2197, \mathrm{DEC}=13.4509)$. The red curves show the surface brightness profile for the best fitting density model.

one with the same center, but PA from $234^{\circ}-273^{\circ}$ and radii $175.1-270.6^{\prime \prime}$. Note that the region from which we extracted a spectrum for outside the south-east front has a slightly larger opening angle compared to the region in which the surface brightness profile was fitted. The sector is wider for better statistical accuracy. Spectra and responses for these regions were extracted as described in $\$ 2.3 .1$

The spectra outside and inside the fronts were fitted successively, with the regions outside the front fitted using an absorbed MEKAL model with the column density and metallicity fixed to the values derived in 2.3.1. The regions inside the fronts were then fitted with a model containing two absorbed MEKAL components, the primary MEKAL component models the gas within the front and the secondary MEKAL component accounts for gas lying in projection along the line of sight, which is assumed to have the same thermal properties as the gas lying in the region outside the front. The column density and abundance were fixed to the values derived in 92.3 .1 for both components and the secondary component had its temperature fixed to the best fit value obtained from the outer region spectra. The normalization of the secondary component was fixed to the value obtained from fitting the spectrum outside the front, corrected by a factor that accounts for the different emission measures expected from the different volumes probed, which is calculated by integration of the density model. For the south-east front, we measured a temperature of $5.7_{-1.7}^{+3.7} \mathrm{keV}$ outside the front and $3.6_{-0.7}^{+1.0} \mathrm{keV}$ inside the front, and for the north-west front we measured a temperature of $5.2_{-1.0}^{+1.5} \mathrm{keV}$ outside the front and $3.2_{-0.7}^{+1.0} \mathrm{keV}$ inside the front.

The pressure should be continuous at a cold front. The pressure jump across a front can be measured by taking the ratio $\left(n_{e, 1} k T_{1}\right) /\left(n_{e, 2} k T_{2}\right)$. We measured pressure jumps of $1.4_{-0.8}^{+1.8}$ and $1.1_{-0.6}^{+1.1}$ for the south-east and northwest fronts, respectively, consistent with the pressure being continuous across both fronts. A shock interpretation for the fronts can be also ruled out by applying the Rankine-Hugoniot shock jump conditions for the measured density jumps and post-shock temperatures (i.e. the temperature on the dense side of the front) and comparing with the values observed. For shock fronts, we would expect to observe pre-shock gas temperatures of $1.9_{-0.4}^{+.9} \mathrm{keV}$ and $2.1_{-0.8}^{+1.2} \mathrm{keV}$ for the south-east and northwest fronts, respectively, significantly different from our measured temperatures. With continuous pressure and a temperature increase, these discontinuities have the hallmarks of cold fronts.

\subsubsection{Temperature Map}

Temperature maps provide extremely useful tools for searching for multi-phase temperature structure due to the effects of an ongoing merger. To search for evidence of merger induced temperature structure in the ICM of Abell 1201, we generated a temperature map using the broad energy band method described in Markevitch et al. (2000). Briefly, we produced source and background images, binned in $7.8^{\prime \prime}$ pixels, in the energy bands 0.5-1.0-2.0-5.0-10 keV, excluding point sources. Exposure maps that corrected for mirror vignetting relative to the on-axis position, QEU (including low energy contamination) and exposure time were produced for each energy band. The background images, which were taken from the blank sky observations described above, were normalized by the ratio of the source to background $9-12 \mathrm{keV}$ counts, subtracted from the source images and the resulting image divided by its corresponding exposure map. Each image was smoothed using the same variable width Gaussian, where $\sigma$ varied from 8.6-39.4", with it being smallest in the brightest regions and chosen such that statistically significant temperatures could be measured whilst maximum spatial information was retained. The noise in each pixel was determined from the raw, uncorrected images and weighted accordingly to allow for the effects of the smoothing.

Each pixel was fitted with an absorbed single temperature MEKAL model with the absorption column set at the Galactic value. The metal abundance was set to the best fitting average cluster value derived in \$2.3.1 The model was multiplied by an on-axis ARF to correct for the energy dependent on-axis mirror effective area, including the chip quantum efficiency (QE). A fluxweighted spectral response matrix was generated from a 


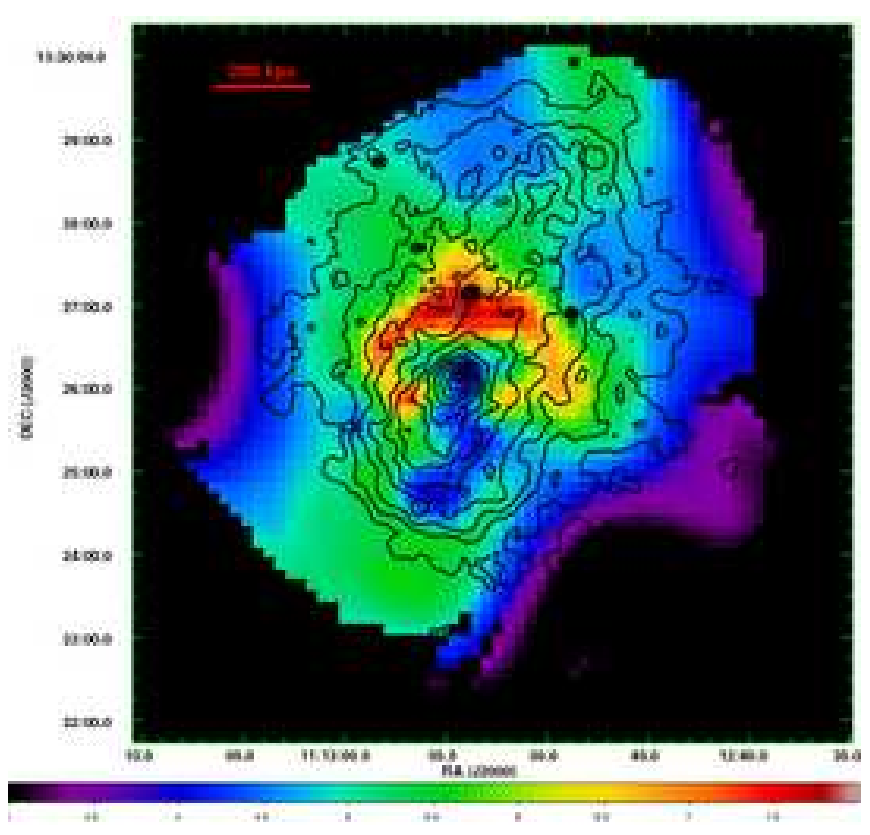

FIG. 6. - Temperature map with $0.5-7.0 \mathrm{keV}$ brightness contours overlaid (same as Figure 2). The $68 \%$ temperature uncertainties range from $\pm 0.4 \mathrm{keV}$ in the cooler $(\mathrm{kT}<5 \mathrm{keV})$ regions to $\pm 1-2$ in the hotter $(\mathrm{kT}>5 \mathrm{keV})$ regions. The color differences represent regions which are at significantly different temperatures. The color bar shows the temperature scale in $\mathrm{keV}$.

large cluster region, and was binned to match the chosen energy bands. The temperature map is presented in Figure 6. where we have excluded pixels where the $1 \sigma$ error is greater than $30 \%$ of the best fit temperature value.

The cold fronts described above are clearly visible in Figure 6] and the temperature map values are consistent with the temperatures measured spectroscopically in the above sections. Interestingly, there is a finger of cool gas extending from the south-east cold front to the core of the cluster. This finger is coincident with the residual observed after subtraction of the Beta model in \$2.2.1 Also of interest is the significantly hotter $\sim 7.5 \mathrm{keV}$ region lying between the core and the excess subclump. The temperature in the region containing the north-west excess appears to be consistent with the global cluster temperature. Since the surface brightness here is low, the photon numbers are also low, meaning the region must be heavily smoothed to obtain statistically significant temperatures $\left(\sigma \simeq 20^{\prime \prime}\right.$ here). Thus the temperature measurement is averaged over a large region and is heavily contaminated by ambient gas projected onto this region, thus the temperature of the excess remains uncertain.

\section{OPTICAL SPECTROSCOPY - REDUCTION AND ANALYSIS}

In this section we present our optical analysis which includes selection of photometric samples for spectroscopic follow up, spectroscopic observations and data analysis, determination of cluster membership and substructure detection. The purpose of this optical analysis is to search for substructures and use them in correlation with the X-ray observations to develop a scenario for the formation of the cold fronts observed above, and also for the merger history of Abell 1201.
The multi-object spectroscopic (MOS) data presented in this paper were taken from two sets of observations, one set at the $3.9 \mathrm{~m}$ Anglo-Australian Telescope (AAT) on 2006 April 2-5, and the second at the $6.5 \mathrm{~m}$ Multiple Mirror Telescope (MMT) in queue schedule mode in the months of February and April 2007. The observation details are listed in Table 3 where we list the dates, magnitude limits, frame exposures and the seeing.

\subsubsection{Parent Photometric Catalog}

The initial target catalog was taken from the Sloan Digital Sky Survey (SDSS) sky server ${ }^{6}$, with all objects within an 18 arcmin radius of the center of Abell 1201 $\left(\mathrm{RA}=11^{\mathrm{h}} 12^{\mathrm{m}} 54.5^{\mathrm{s}}, \mathrm{DEC}=+13^{\circ} 26^{\prime} 09.0^{\prime \prime}\right)$ included. This catalog was then filtered to include only those objects classified by the SDSS pipeline as galaxies (SDSS class 3 ). The SDSS $r$ and $g$ magnitudes for the remaining objects were then converted to Johnson $B$ and $R$ magnitudes via equations A5 and A7 of Cross et al. (2004) and only those galaxies with $\mathrm{R}<21.5$ were retained in the final target list.

The target galaxies were then ranked based on their cluster-centric radius and their position on the B-R vs $\mathrm{R}$ color-magnitude (CM) diagram, in preparation for the fiber allocation procedure. Galaxies on or blueward of the red sequence are more likely to be cluster members and so were ranked higher than those redward of the red sequence. Those galaxies either on or blueward of the red sequence were then ranked by cluster-centric distance, with those closest to the center ranked highest. The remaining galaxies lying redward of the red sequence were also ranked by cluster-centric distance. These rankings were input into the software used to configure the fiber allocations for the respective observations, to ensure that higher weightings were placed on galaxies which were more likely to be cluster members (i.e. those on and blueward of the red sequence and closer to the center). We discuss the configuration process further below.

We show the CM diagram in Figure 7, where we have overplotted the spectroscopically confirmed cluster members (green stars), foreground galaxies (blue squares) and background galaxies (red circles). Also plotted is the line $B-R=3.6-0.0645 R$ which was used to distinguish those galaxies which we define to be lying on or below the red sequence from those redward of the red sequence. The slope of the line is calculated from the best fit to the CM slope versus redshift diagram of López-Cruz et al. (2004) and the constant is estimated by eye such that the line lies just above the red sequence.

\subsubsection{AAT AAOmega Observations}

The AAT observations were taken using the AAOmega fiber-fed spectrograph (Saunders et al. 2004; Smith et al. 2004; Sharp et al. 2006), which is a bench mounted dualbeam spectrograph, which is fed by 400 fibers roboticallyplaced within the two degree field at the telescope's prime focus. A total of 392 fibers, each $2^{\prime \prime}$ in diameter, are available for the simultaneous observation of scientific targets (with the remaining 8 fibers being used for acquisition and guiding). All our observations were taken using the medium resolution $(R \simeq 1300) 580 \mathrm{~V}$ (blue arm) and 385R (red arm) gratings, which in combination with

\footnotetext{
6 See: http://cas.sdss.org/dr5/en/ 
TABLE 3

Summary of the obSERVATions.

\begin{tabular}{|c|c|c|c|c|}
\hline Telescope/Instrument & Date & Magnitude & Frames & Seeing \\
\hline AAT/AAOmega & 2006 Apr 2 & $19.5<R<20.5$ & $\begin{array}{c}3 \times 1500 \mathrm{~s}+ \\
3 \times 750 \mathrm{~s}\end{array}$ & $1.5-2.0^{\prime \prime}$ \\
\hline . & 2006 Apr 4 & $19.5<R<20.5$ & $3 \times 1800 \mathrm{~s}$ & $2.0^{\prime \prime}$ \\
\hline$\ldots$ & 2006 Apr 5 & $R<19.5$ & $3 \times 1200 \mathrm{~s}$ & $2.0^{\prime \prime}$ \\
\hline$\ldots$ & 2006 Apr 5 & $R<19.5$ & $3 \times 1200 s$ & $2.0^{\prime \prime}$ \\
\hline$\cdots$ & 2006 Apr 5 & $R<19.5$ & $3 \times 1200 s$ & $2.5^{\prime \prime}$ \\
\hline MMT/Hectospec & 2007 Feb 21 & $20.5<R<21.5$ & $4 \times 1800 s$ & $0.6^{\prime \prime}$ \\
\hline$\ldots$ & 2007 Feb 22 & $20<R<20.5$ & $4 \times 1200 s$ & $1.2^{\prime \prime}$ \\
\hline$\ldots$ & 2007 Apr 18 & $R<20.5$ & $3 \times 1200 s$ & $1.2^{\prime \prime}$ \\
\hline$\cdots$ & 2007 Apr 19 & $R<20.5$ & $4 \times 1200 \mathrm{~s}$ & $0.8^{\prime \prime}$ \\
\hline
\end{tabular}

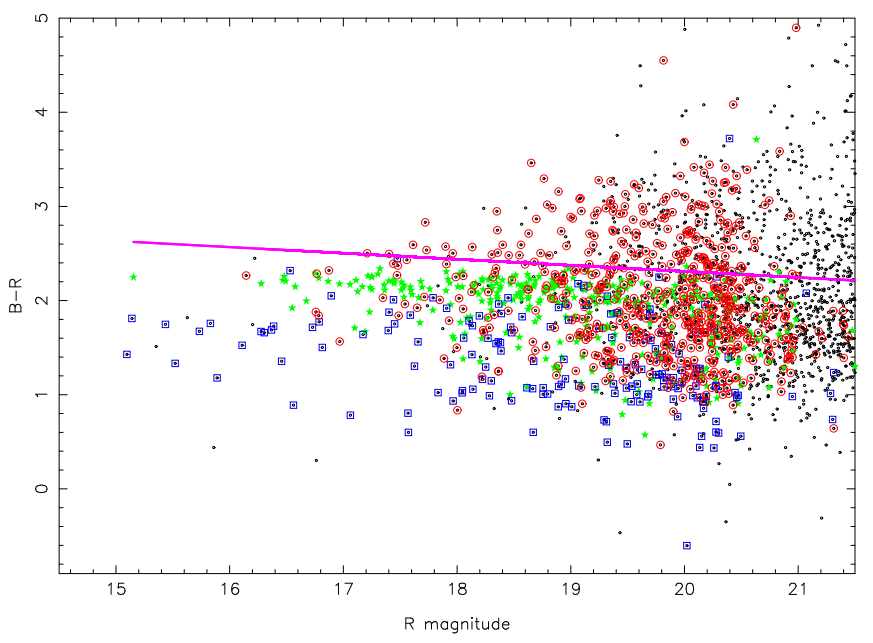

Fig. 7.- Color-magnitude diagram for all galaxies within our observed field (black dots). The green stars represent cluster members, the blue squares foreground galaxies, and the red circles background galaxies. The pink line is the line used to delineate galaxies on and blueward of the red sequence from those redward of the red sequence for the purpose of ranking galaxies during fiber configuration.

the $15 \mu \mathrm{m}$ pixel $2 \mathrm{k} \times 4 \mathrm{k} \mathrm{E} 2 \mathrm{~V}$ CCD detectors produce a spectral resolution of $3.6 \AA$ and $5.5 \AA$ in the blue and red arms, respectively, and provide an overall wavelength coverage ranging from 3700 to $8800 \AA$.

Due to the clustered nature of the galaxies, fiber positioning constraints (minimum separation of $\sim 30^{\prime \prime}$ due to the physical size of the fiber buttons) and the fact our input catalog contained $\sim 1400$ galaxies, multiple plate configurations were required to obtain high overall spectral completeness and adequate coverage in the central regions of the cluster. The fibers were allocated using the AAOmega CONFIGURE software ${ }^{7}$. A total of 5 configurations were required for Abell 1201: three for galaxies with $R<19.5$, and two for galaxies in the range $19.5<R<20.5$ plus those that remained unallocated in the three 'bright' configurations. Due to packing constraints, only $110-210$ of the available fibers were allocated to science objects. In addition, 30-40 fibers were allocated to blank sky regions for sky subtraction. All fibers known to suffer from interference fringing effects were left unallocated. The details for each configuration observation are listed in Table 3 . The observations were taken in rather mediocre conditions, with the seeing (FWHM) ranging from 1.5 to 2.5 arcsec, and all ex-

\footnotetext{
7 See: http://www.aao.gov.au/AAO/2df/
}

posures being taken through thin cloud cover, with some being interrupted by thicker clouds. In commencing the observations for each new configuration, a $4 \mathrm{~s}$ dome flat and $60 \mathrm{~s}$ FeAr arc lamp exposure were taken for flat fielding and wavelength calibration of the data, respectively. The data were reduced with the AAO $2 d F D R$ pipeline software with a patch included to fix fiber to slit position mapping errors present in AAOmega data taken before August 2006.

Redshift identification and measurement for each spectrum was carried out using the RUNZ code written by Will Sutherland for the 2dF Galaxy Redshift Survey (2dFGRS; Colless et al. 2001). This program utilizes the cross-correlation method of Tonry \& Davis (1979), based on a library of galaxy template spectra that are representative of all the different observed spectral types. Each spectrum was inspected visually and given a redshift quality classification, Q. Here we used the same scheme as adopted for the 2dFGRS, with each spectrum being assigned a $Q$ value on a six-point integer scale, with $\mathrm{Q}=1$ indicating that no redshift could be estimated, $\mathrm{Q}=2$ a possible but unreliable redshift, $\mathrm{Q}=3$ a probable redshift (with $\sim 90 \%$ confidence), $\mathrm{Q}=4$ a reliable redshift, $\mathrm{Q}=5$ a reliable redshift with high-quality spectrum, and $\mathrm{Q}=6$ indicating a star or non-extragalactic object. We obtained spectra for 917 galaxies during the run, which yielded reliable $(\mathrm{Q}=3,4$, or 5$)$ redshift measurements for 580 single galaxies.

\subsubsection{MMT Hectospec Observations}

The MMT observations were taken using the Hectospec multi-object spectrograph (Fabricant et al. 2005). This is also a bench-mounted spectrograph, which is fed by $3001.5^{\prime \prime}$ diameter fibers that cover a 1 degree field of view. The observations were taken using the medium resolution $(\mathrm{R}=1000-2000) 270$ groove $\mathrm{mm}^{-1}$ grating and the data were captured on a single array of two E2V CCDs with $13.5 \mu \mathrm{m}$ pixels, resulting in spectra with a resolution of $6.2 \AA$ and covering the wavelength range $3500-10000 \AA$.

For the MMT observations, we included any galaxy not observed during the AAT run, as well as galaxies that were not assigned reliable redshifts. A small number of galaxies which were assigned a redshift quality of $\mathrm{Q}=3$ (from their AAT spectra) and were in the redshift range $0.15-0.18$ (i.e. close to the cluster in redshift space), were also included in order to check on our redshift accuracy. Fiber configurations were generated using the XFITFIBS 
software ${ }^{8}$, with a total of four being required for galaxies with $R<20.5$, and one being required for galaxies with $20.5<R<21.5$. Typically 150 fibers were allocated to galaxies, with around 100 fibers allocated to blank sky areas. The details of each observed configuration are listed in Table 3 . The seeing during the observations ranged from $0.61-1.2^{\prime \prime}$, and the observations performed in the worst seeing conditions were somewhat affected with a number of galaxies requiring re-observation since the spectra were inadequate for determining redshifts. The data were reduced at the Telescope Data Center (TDC) ${ }^{9}$ using the TDC pipeline. The data were also redshifted at the TDC using the IRAF cross-correlation XCSAO software (Kurtz et al. 1992) and spectra were assigned a redshift quality of "Q" for reliable, "?" for questionable and "X" for bad redshift measurements. We use in our analysis only those galaxies with reliable redshift measurements (quality=Q). A subsample of the data was visually inspected and the redshifts and quality assignments were found to be robust. We obtained spectra for 742 galaxies during the run, which yielded reliable redshift measurements for 534 single galaxies.

\subsubsection{Redshift completeness and measurement errors}

A number of galaxies were observed multiple times in the two observing runs. There were also 67 galaxies with independently measured redshifts within 20 arcmin of the cluster center in the NASA Extragalactic Database $(\mathrm{NED})^{10}$. To check the accuracy of our measured redshifts, we compared the redshifts of all multiply-observed galaxies by determining the mean of the differences as well as their standard deviation. The MMT observations had a mean intrinsic uncertainty of $42 \pm 1 \mathrm{~km} \mathrm{~s}^{-1}$ for 534 redshifts. There were 19 repeat observations made with the MMT, and the mean difference was $1 \pm 25 \mathrm{~km} \mathrm{~s}^{-1}$ after removing a spurious result where the difference was $c z=44894 \mathrm{~km} \mathrm{~s}^{-1}$. From these repeat observations, we calculate an RMS of $105 \mathrm{~km} \mathrm{~s}^{-1}$ implying an uncertainty of $74 \mathrm{~km} \mathrm{~s}^{-1}$ for the MMT observations, higher than the mean of the individual redshift measurements. An external test of these redshift uncertainty measurements comes from the 12 redshifts in common between the NED and MMT catalogs which have a mean difference of $-28 \pm 20 \mathrm{~km} \mathrm{~s}^{-1}$ with an RMS scatter of $74 \mathrm{~km} \mathrm{~s}^{-1}$. The NED redshifts come primarily from the SDSS and the catalog of Miller et al. (2002) which have redshift uncertainties $\sim 30 \mathrm{~km} \mathrm{~s}^{-1}$. Taking this into account gives an external uncertainty measurement of $43 \mathrm{~km} \mathrm{~s}^{-1}$, consistent with the mean of the individual uncertainty measurements. The high uncertainty value given by the repeat MMT observations can be understood by considering that these objects were re-observed due to the low quality of their initial redshift measurements.

There were no repeat observations taken at the AAT, which have a mean internal uncertainty for the individual redshift measurements of $109 \pm 6 \mathrm{~km} \mathrm{~s}^{-1}$ from 580 redshifts. An external check of this value comes from 31 galaxies re-observed with the MMT which had both $\mathrm{Q}=3$ redshifts from the AAT data set and quality $=\mathrm{Q}$ from the MMT. The mean difference in the measured

\footnotetext{
8 see:http://cfa-www.harvard.edu/mmti/hectospec.html

9 see: http://tdc-www.harvard.edu/

10 see:http://nedwww.ipac.caltech.edu/
}

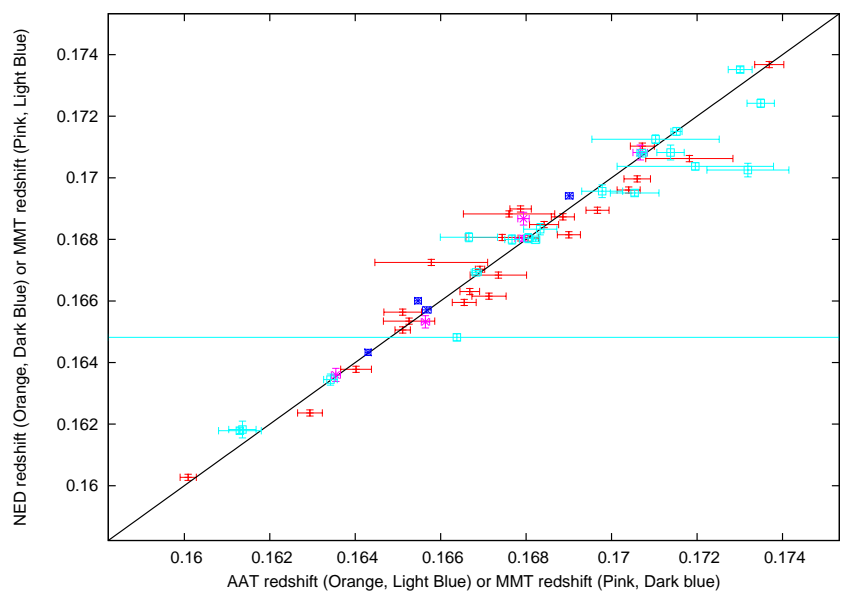

FIG. 8.- Redshift comparison for Abell 1201 using our MMT and AAT redshifts, and also a sample of NED redshifts. The comparison of different measurements are plotted with the following color codes: $\mathrm{MMT} / \mathrm{MMT}=$ pink, AAT $/ \mathrm{MMT}=$ light blue, AAT $/ \mathrm{NED}$ $=$ orange, $\mathrm{MMT} / \mathrm{NED}=$ dark blue. The black line represents the one-to-one relationship; the general scatter of the data about this line indicates the absence of any gross systematic errors in our measurements, and indicates our AAT and MMT measurements have a precision of $159 \mathrm{~km} \mathrm{~s}^{-1}$ and $74 \mathrm{~km} \mathrm{~s}^{-1}$, respectively. We assume an uncertainty of $30 \mathrm{~km} \mathrm{~s}^{-1}$ for the NED measurements.

redshifts of these was $50 \pm 44 \mathrm{~km} \mathrm{~s}^{-1}$. Two galaxies with obviously spurious redshift differences of $\Delta(c z)=$ $53417 \mathrm{~km} \mathrm{~s}^{-1}$ and $\Delta(c z)=119800 \mathrm{~km} \mathrm{~s}^{-1}$ were excluded from this calculation. The RMS scatter in the difference was $248 \mathrm{~km} \mathrm{~s}^{-1}$, and taking into account the uncertainty derived above for the MMT redshifts $\left(74 \mathrm{~km} \mathrm{~s}^{-1}\right)$, we calculate an uncertainty of $159 \mathrm{~km} \mathrm{~s}^{-1}$. This value is higher than the mean of the individual redshift uncertainties, however again it is noted that these objects were re-observed due to their poor initial redshift measurements (all had $\mathrm{Q}=3$ ), thus the measured uncertainty is expected to be higher. This value can be checked using the 45 measurements in common with the NED catalog, where the mean difference was $33 \pm 29 \mathrm{~km} \mathrm{~s}^{-1}$, and an RMS scatter in the differences of $191 \mathrm{~km} \mathrm{~s}^{-1}$, after two spurious results where the redshift differences were $\Delta(c z)=3480 \mathrm{~km} \mathrm{~s}^{-1}$ and $\Delta(c z)=10472 \mathrm{~km} \mathrm{~s}^{-1}$ were excluded. Based on these redshift differences, we measure an uncertainty of $132 \mathrm{~km} \mathrm{~s}^{-1}$, again after accounting for the $\sim 30 \mathrm{~km} \mathrm{~s}^{-1}$ uncertainty associated with the NED redshifts.

Figure 8 shows the comparison of redshift measurements using different instruments in the redshift range of the cluster. The figure, in combination with the redshift differences above, show that there are no gross systematic errors in our measurements and the scatter about a 1-to1 relationship is well encompassed by the uncertainties of $159 \mathrm{~km} \mathrm{~s}^{-1}$ and $74 \mathrm{~km} \mathrm{~s}^{-1}$ for AAT and MMT, respectively, which we take from the more conservative higher values derived above from the double observations, and these values indicate the precision of our measurements.

For galaxies which had multiple redshift measurements, the following approach was taken to determining what their final adopted redshift would be: If a galaxy was observed with both the MMT and AAT, we used the MMT redshift since the MMT redshifts were in general more precise with lower errors (as expected since the reobserved galaxies were ones with AAT quality $\mathrm{Q}=3$ ). If a galaxy was observed more than once with the MMT, we 
took the redshift based on the highest cross-correlation coefficient. If a galaxy was observed more than once with the AAT, we took the redshift with the highest $\mathrm{Q}$ value. Note that in no cases did we ever use the NED redshift if that galaxy had been observed on either the AAT or MMT. When added to our single redshift measurements, this yielded a total of 560, 534 and 10 redshifts measured with the AAT, MMT and sourced from NED, respectively, giving an overall total of 1104 robust redshifts acquired within the cluster field.

We determined the spectroscopic completeness of the sample by measuring the ratio of galaxies in the parent photometric catalog which have reliable redshifts (as defined previously) to those which have no reliable redshift measurement. The spectroscopic completeness as a function of cluster-centric radius for the $R$ magnitude intervals $0-18,18-19.5,19.5-20.5$, and $20.5-21.5$ is plotted in Figure 9 where we also plot the spectroscopic completeness within $3.5 \mathrm{Mpc}$ as a function of $R$ magnitude. We achieve $\gtrsim 80 \%$ spectroscopic completeness at all radii, apart from within the $3-3.5 \mathrm{Mpc}$ range, for magnitudes brighter than 19.5 , whilst we obtain $\sim 60 \%$ spectroscopic completeness at all radii for magnitudes between 19.5 and 20.5 and $\sim 20 \%$ spectroscopic completeness for magnitudes between 20.5 and 21.5 within a radius of $\sim 2.5 \mathrm{Mpc}$. Thus our catalog is very well sampled at all radii for $R$ magnitudes brighter than 20.5, which corresponds to $\sim 2.3$ mags down the cluster luminosity function (assum$\operatorname{ing} M_{R}^{*}=-21.3$ (Yagi et al. 2002), and neglecting galactic extinction and K-correction terms).

\subsection{Cluster member selection}

Identification of cluster members from our spectroscopic redshifts was achieved through the elimination of foreground and background galaxies along the line of sight to Abell 1201. An initial rejection was performed using the "velocity gap" method outlined by De Propris et al. (2002), where the galaxies are sorted in redshift space and the velocity $(c z)$ gap between each one determined. Here the velocity gap for the nth galaxy is $\Delta v_{n}=c z_{n+1}-c z_{n}$. Clusters appear as well populated peaks in redshift space which are separated by velocity gaps of greater than $1000 \mathrm{~km} \mathrm{~s}^{-1}$ from the nearest foreground and background galaxies, and Figure [10] shows that Abell 1201 is readily identified on this basis.

Due to the filamentary structures which surround clusters, it is necessary to further refine this rejection process. For this refinement, we used a slightly different version of the "shifting gapper" method first implemented by Fadda et al. (1996) where both cluster-centric radius and peculiar velocity information are used and the gap method outlined above is applied as a function of radius. The galaxies were binned radially such that each bin contained 35 galaxies, and were then sorted by $v_{p e c}$, with the velocity gaps determined as before (but in peculiar velocity, not $c z$ ). Peculiar velocities were determined with respect to the central cluster redshift using the following procedure: We estimated the mean cluster redshift using the biweight location estimator (Beers et al. 1990) which we assume represents the cosmological redshift of the cluster. The peculiar redshift, $z_{\text {pec }}$, was derived under the assumption that the observed redshift of the galaxy, $z_{\text {gal }}$, is comprised of only two components, the cosmological component, $z_{c o s}$, and the component due to the pecu- liar motion within the cluster, $z_{p e c}$. Hence, the peculiar redshift is $z_{p e c}=\left(z_{\text {gal }}-z_{\text {cos }}\right) /\left(1+z_{\text {cos }}\right)$ and the peculiar velocity was derived using the standard special relativistic formula $v_{p e c}=c\left(\left(1+z_{p e c}\right)^{2}-1\right) /\left(\left(1+z_{p e c}\right)^{2}+1\right)$, where $c$ is the speed of light.

We used the "f pseudosigma" (Beers et al. 1990), derived from the first and third quartiles of the peculiar velocity distribution, as the fixed gap to separate the cluster from interlopers. The f pseudo-sigma is an estimator of the scale of the velocity distribution which is robust to the presence of velocity interlopers in the tails of the cluster distribution. The above procedure was iterated until the number of members was stable and the results are shown in Figure 11 where it can be seen that the cluster is clearly separated from the filamentary structure surrounding it, and the interlopers are cleanly rejected. The advantage of this method is that it does not assume a particular mass model or velocity distribution which both rely on the cluster being relaxed.

The final cluster sample contains 321 members out to a cluster-centric radius of $\sim 3.5 \mathrm{Mpc}$. The final value for the biweight location estimator of the cluster redshift is $z_{\text {cos }}=0.1673 \pm 0.0002$. We use the biweight scale estimator (Beers et al. 1990) to estimate a velocity dispersion of $778 \pm 36 \mathrm{~km} \mathrm{~s}^{-1}$. The errors for the redshift and velocity dispersion are $1 \sigma$ and are estimated using the jackknife resampling technique.

\subsection{Substructure Detection}

Now that we have separated our cluster members from foreground and background interlopers, we can apply substructure detection tests to the cluster member sample. Substructure in a cluster can present itself in a number of different ways, and generally no single statistical test is capable of revealing all such manifestations. Hence it is essential that the full range of statistical tests are applied if the search for substructure is to be an exhaustive one. In general, statistics using the maximum amount of information (eg., the radial velocity and spatial dimensions) are the most effective at revealing substructure, however these can fail if, for example, roughly equal mass clusters are merging along the line of sight such that the cores are spatially coincident (see eg. Girardi et al. 2006; Pinkney et al. 1996). The use of velocity information by itself can be successful, although there are documented cases where the velocity distribution mimics that of a relaxed cluster where other methods have clearly shown it to be disturbed (Maurogordato et al. 2000; Johnston-Hollitt et al. 2008). The use of 2-D spatial information alone suffers from foreground and background contamination. In this section, we apply a number of statistical methods to the detection of substructure in Abell 1201.

\subsubsection{Deviations from Gaussianity}

The velocity distribution of a relaxed cluster is well approximated by a single Gaussian, with velocity dispersion related to the cluster mass via the virial theorem. Departures from Gaussianity can be attributed to a dynamically active cluster where substructures may cause symmetric and asymmetric distortions in the velocity distribution. As a first test of Gaussianity, we used the standard Kolmogorov-Smirnov (K-S) test. At the $90 \%$ confidence level, the observed velocity distribution 

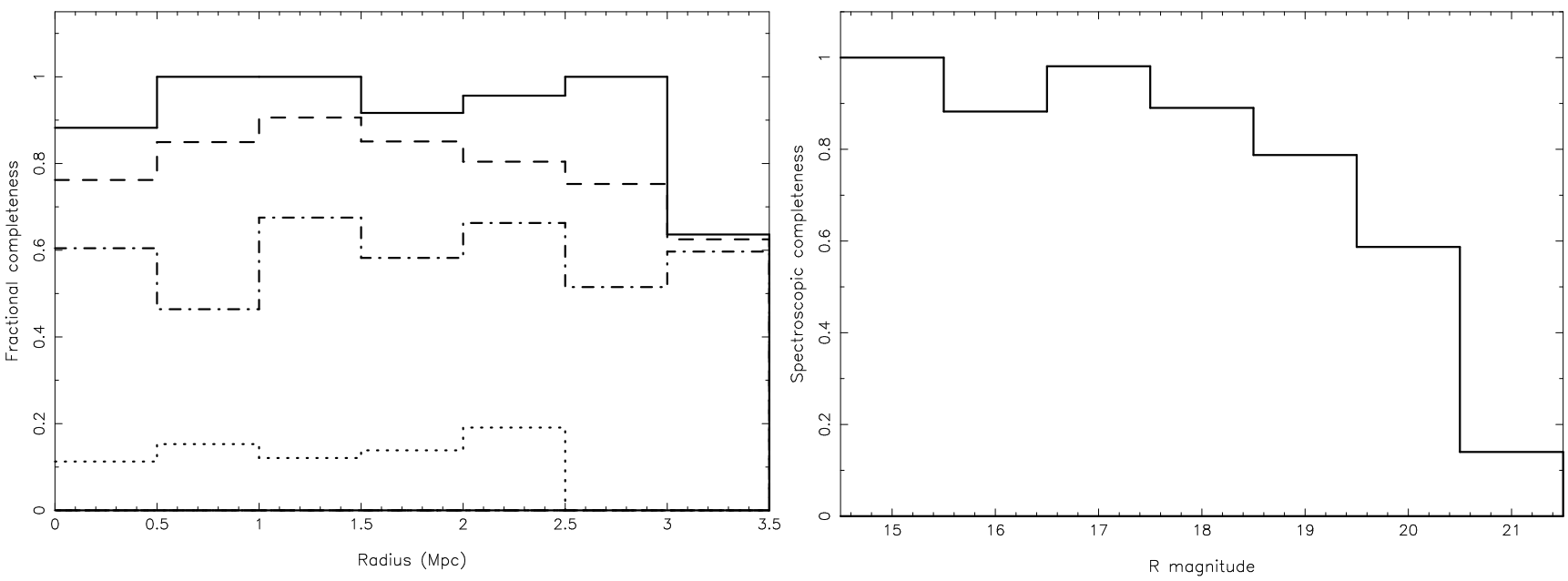

FIG. 9.- Spectroscopic completeness as a function of cluster-centric radius (left panel) for the $R$-band magnitude intervals 0 -18 (solid line), 18-19.5 (dashed line), 19.5-20.5 (dot-dashed line), and 20.5-21.5 (dotted line). This shows we have both good completeness and radial coverage for magnitudes brighter than $R=20.5$. The spectroscopic completeness as a function of magnitude is shown in the right panel.

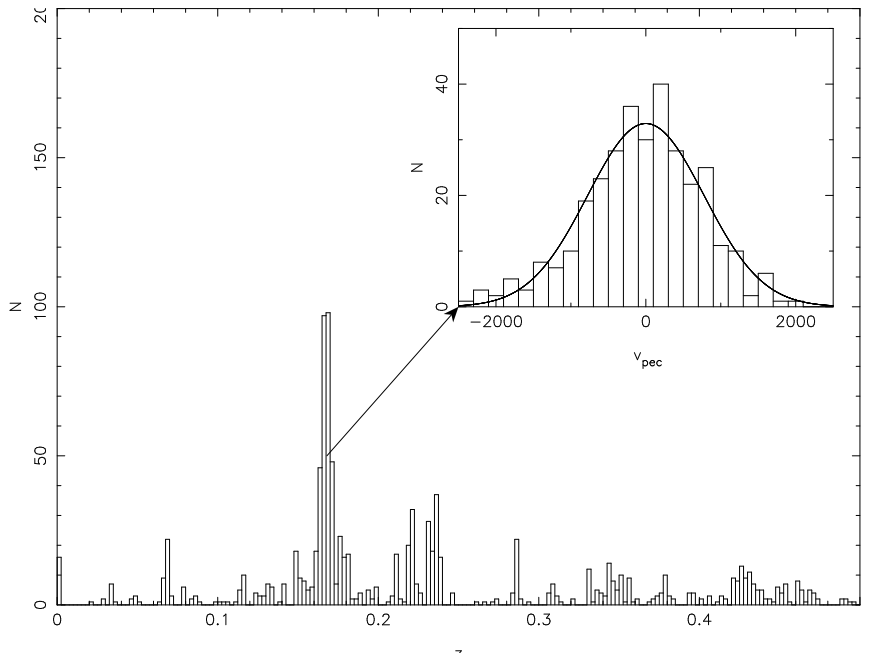

FIG. 10.- A histogram of all the reliable redshifts measured within the Abell 1201 field. The Abell 1201 cluster clearly stands out against the foreground and background galaxies. The inset panel shows the distribution of peculiar velocities for the cluster members, with a Gaussian with mean zero and standard deviation of $778 \mathrm{~km} \mathrm{~s}^{-1}$ overplotted.

is consistent with being drawn from a Gaussian distribution with $\overline{v_{p e c}}=0$ and $\sigma_{v p e c}=778 \mathrm{~km} \mathrm{~s}^{-1}$. On the basis of this test, therefore, it appears unlikely Abell 1201's velocity distribution is significantly non-Gaussian.

The disadvantage of using the $\mathrm{K}-\mathrm{S}$ test is that it is most sensitive to the behavior of the distribution near its median, but is relatively insensitive to differences in the tails of the distribution. Also, the K-S test does not give quantitative information about the way in which two distributions differ. Quantifying these deviations is imperative in determining the state of the cluster, and a number of different methods can be used to quantify asymmetric and symmetric distortions (Pinkney et al. 1996). Here we choose to use the method outlined in Zabludoff et al. (1993), where the velocity distribution, $L$, is approximated by a series of Gauss-Hermite functions

$$
L=\sum_{j=0}^{4} h_{j} H_{j}(x)
$$

where $h_{j}$ are the Gauss-Hermite moments defined by

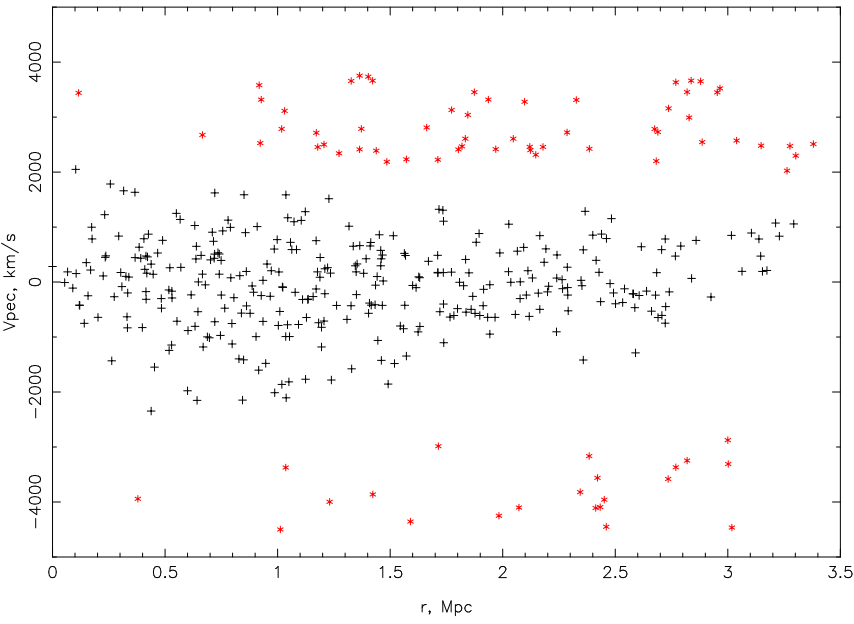

FIG. 11.- A plot of deviations in peculiar velocities as a function of cluster-centric radius, illustrating the efficacy of our refined rejection method whereby foreground and background interlopers are identified and eliminated using a shifting gapper technique (see text). The black crosses represent galaxies allocated as cluster members while the orange asterisks are rejected foreground and background galaxies lying close to the cluster in redshift space.

Zabludoff et al. (1993) as

$$
h_{j}=\frac{2 \sqrt{\pi}}{N S} \sum_{i=1}^{N} H_{j}\left(x_{i}\right),
$$

where

$$
x_{i}=\frac{v_{p e c, i}-V}{S}, H_{j}(x)=\frac{e^{-x^{2} / 2}}{\sqrt{2 \pi}} \mathcal{H}_{j}(x),
$$

and $\mathcal{H}_{i}(x)$ are the Hermite polynomials given by van der Marel \& Franx (1993). In principle, the velocity distribution can be described by equations 4 and 5 and a wide variety of $S$ and $V$ values, where there is significant degeneracy in the choices of $S$ and $V$ and the $j=1,2$ terms. However, as pointed out by van der Marel \& Franx (1993), the most efficient method is to set $S$ and $V$ so that the zeroth-order term, $H_{0}$, describes the best fit Gaussian to the data. This is achieved by choosing $S$ and $V$ such that $h_{1}=h_{2}=0$. We iterated 
with different values of $S$ and $V$ until these criteria were met. The terms $h_{3}$ and $h_{4}$ describe, respectively, the asymmetric and symmetric deviations from Gaussianity, much like the higher order Gaussian skewness and kurtosis terms, but have the advantage of being less sensitive to outliers in the tails of the distribution. Applying this method to the velocity distribution for Abell 1201, we obtain values of values of $h_{3}=-0.016$ and $h_{4}=0.023$. We used 10,000 Monte Carlo realizations of Gaussian distributions with $\mathrm{N}=321, \sigma=S$ and $\mu=V$ to determine the probability of detecting our $h_{3}$ and $h_{4}$ terms. Values of $\left|h_{3}\right| \geq 0.016$ occur in $70 \%$ of the realizations, while values of $\left|h_{4}\right| \geq 0.023$ occur in $50 \%$ of the realizations. We conclude there is no significant evidence for asymmetric or symmetric deviations from Gaussianity.

\subsubsection{Velocity Distribution Profiles}

Figure 12 shows integral and differential projected radial profiles of the biweight location, $\mu(R)$ and scale, $\sigma(R)$, estimators. The differential profiles are binned using the same radial intervals as used for the shift gapper in \$3.2. such that each bin contains at least 35 data points. To measure the integrated profiles, we sorted the data by cluster-centric radius and for each galaxy in the sample, starting from the tenth, we measured the biweight location and scale (where the number of galaxies was less than 15, we used the median and gapper estimates of location and scale) using only data within the radius of interest to each galaxy.

The integrated and differential $\mu(R)$ are constant with radius within the $1 \sigma$ error bars. This is expected for an isotropic distribution of radial velocities. The differential $\sigma(R)$ profile is flat out to $r=1 \mathrm{Mpc}$, and smoothly declines at larger radii. den Hartog \& Katgert (1996) classified $\sigma(R)$ profiles as flat, inverted or peaked, depending on the shape of the profile within $\sim 1 \mathrm{Mpc}$, and they note an interesting subset of clusters with flat $\sigma(R)$ profiles which have the highest $\sigma(R)$ value measured between 0.5 and $1.0 \mathrm{Mpc}$, all of which show signs of recent merger activity. While this is by no means a definitive sign of merger activity, it is interesting to note that Abell 1201 also exhibits its highest value of $\sigma(R)$ at $\sim 1 \mathrm{Mpc}$. The smooth decline outside of $1 \mathrm{Mpc}$ is expected since the escape velocity of a cluster declines with radius, while radial orbits of infalling galaxies enhance the decline. The integral $\sigma(R)$ profile increases gradually to its maximum value at $\sim 1 \mathrm{Mpc}$, then steadily decreases before flattening out at a radius $\sim 2.5 \mathrm{Mpc}$, indicating the $\sigma$ measured at this radius is no longer affected by velocity anisotropy, and is representative of the cluster potential (Fadda et al. 1996).

We include the estimate of the velocity dispersion derived from the cluster $\mathrm{X}$-ray temperature measured in 2.3 .1 by assuming $\beta=\mu m_{p} \sigma^{2} / k T=1$ (i.e., assuming the same specific kinetic energy in the gas and galaxies), where $\sigma$ is the galaxy velocity dispersion, $\mu$ is the mean molecular weight of the gas particles, $m_{p}$ is the proton mass and $k T$ is the gas temperature within $R \simeq 500 \mathrm{kpc}$. The integral and differential $\sigma(R)$ profiles appear, to within the errors, to be consistent with the value estimated from the X-ray temperature when considering regions within $1 \mathrm{Mpc}$.

\subsubsection{3-D tests for sub-structure}

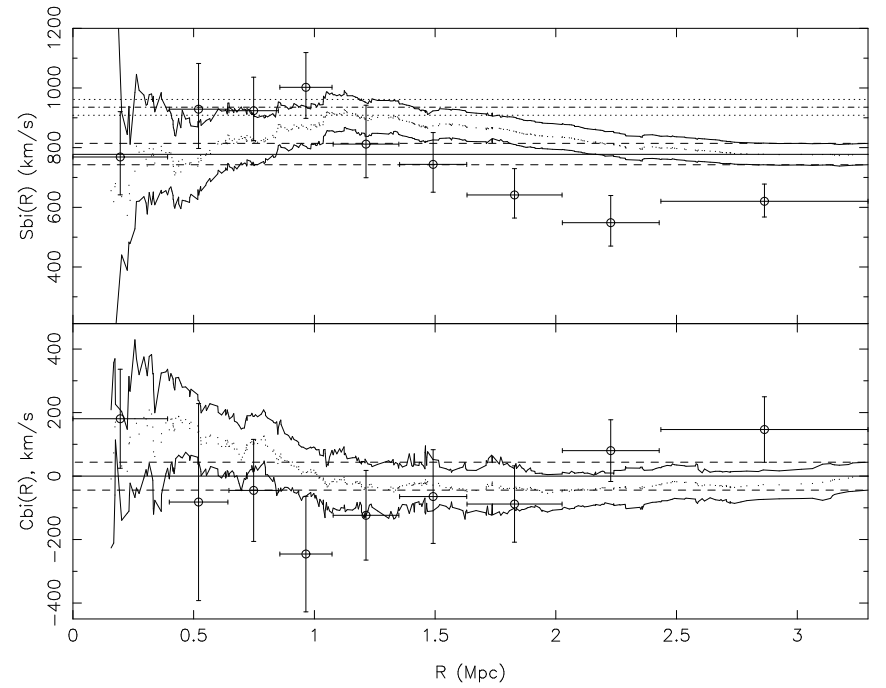

Fig. 12.- Differential velocity dispersion profile (top panel) and mean peculiar velocity (bottom panel) profiles as a function of projected radius. The solid lines show the total cluster values, while the dashed lines and error bars show the $1 \sigma$ confidence limits derived using the jackknife technique. The dot-dashed line shows the velocity dispersion derived from the mean X-ray temperature (see text), with the dotted lines showing the upper and lower limits based on the errors in the temperature (see $\S 2.3 .1$.

The most efficient way to detect real physical substructures is to use a combination of the 2-D spatial and 1-D velocity information to search for local variations in the velocity distribution (Pinkney et al. 1996). The most common method here is to utilize the $\Delta$ statistic developed by Dressler \& Shectman (1988), which tests for differences in the local mean and dispersion compared to the global mean and dispersion. The downfall of this method is that it assumes the global and local peculiar velocity distributions are Gaussian, which may not be true for dynamically active systems. Here we prefer the k-statistic, $\kappa$, employed by Colless \& Dunn (1996), which is very similar to the $\Delta$ statistic but does not require the assumption of Gaussianity to be made. To determine $\kappa$, the $n=\sqrt{N}$ nearest neighbors in projection are selected for each cluster member, where $N$ is the total cluster member sample size, and the local velocity distribution of the $n$ nearest neighbors is compared to the global cluster velocity distribution (minus the $n$ nearest-neighbor velocities). Local departures from the global velocity distribution are quantified using the K-S D statistic, with the null hypothesis that the local distribution is drawn from the global one and the significance determined by measuring the probability that the D statistic is larger than the observed D statistic for the observed sample size, $P_{K S}\left(D>D_{o b s}\right)$. Then, $\kappa_{n}$ is defined as

$$
\kappa_{n}=\sum_{i=1}^{N}-\log P_{K S}\left(D>D_{o b s}\right),
$$

giving a global measure of the substructure present in the cluster by summing the individual $\kappa$ values. The significance of $\kappa_{n}$ was determined by performing 10,000 Monte Carlo realizations with the peculiar velocities randomly shuffled, whilst maintaining the positional information, and remeasuring $\kappa_{n}$.

The observed value of $\kappa_{n}=379$, is larger than any value obtained in our 10,000 realizations, which follow a log-normal distribution with a mean value of 


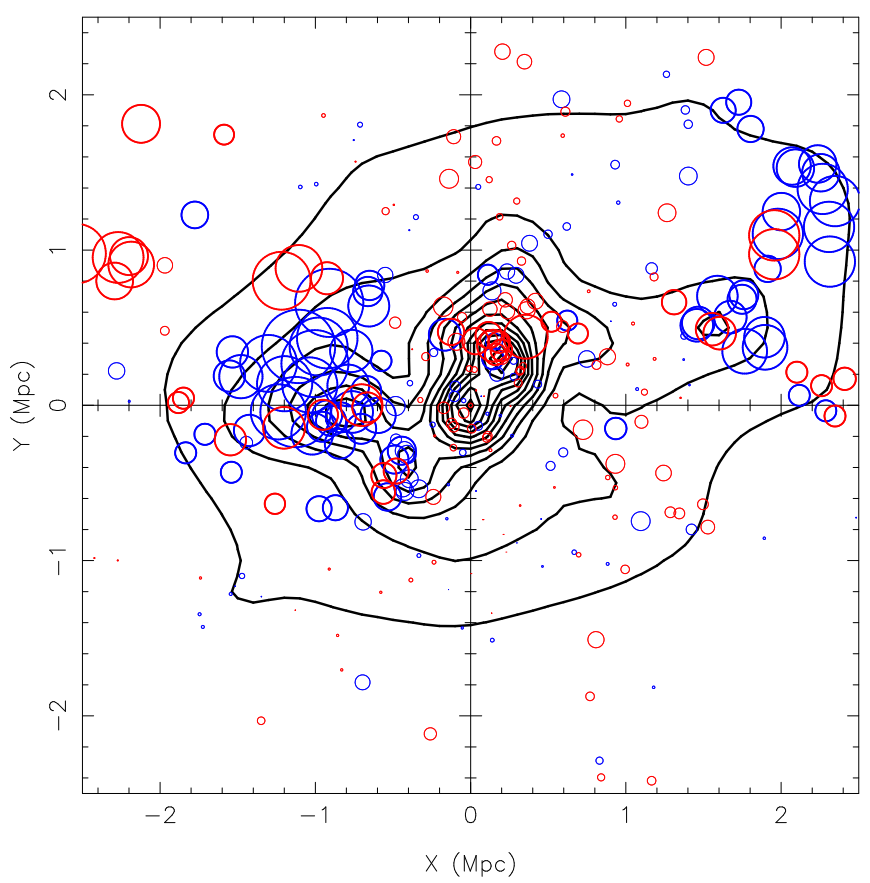

FIG. 13. - Bubble plot outputs from the $\kappa$ test. The bold bubbles are those deemed to be significant insomuch as they only occur in $5 \%$ of 10,000 realizations. The blue bubbles are those galaxies which have negative $v_{p e c}$, and the red bubbles have positive $v_{p e c}$. The contours are galaxy density contours generated from applying a variable width Gaussian filter to the spatial distribution of spectroscopically confirmed members. The contours are linearly spaced by 10 in the interval $10-150$ gals $\mathrm{Mpc}^{-2}$. Note the four clumpings of significant bubbles are coincident with overdensities in the projected galaxy density. The center of the cluster is located at 0,0 Mpc.

$\mu\left(\ln \kappa_{\text {sim }}\right)=4.9$ and standard deviation $\sigma\left(\ln \kappa_{\text {sim }}\right)=$ 0.2 . Thus, the observed $\kappa_{n}$ lies $6 \sigma$ from the mean of the realizations, and we can put an upper limit on the probability of observing this $\kappa_{n}$ value by chance at less than $10^{-4}$. We conclude, therefore, that there is velocity substructure present in Abell 1201 at high significance. The results of the $\kappa$ test are best presented using "bubble plots" showing a circle with radius $r \propto-\log P_{K S}\left(D>D_{o b s}\right)$ at each galaxy position, such that clustered large bubbles reveal local departures from the global velocity distribution. We show these bubbles in Figure 13 and color code them based on the sign of the peculiar velocity, with blue and red having negative and positive $v_{p e c}$, respectively. Overplotted are contours of galaxy surface density which have been produced by applying a variable width Gaussian filter, with $\sigma$ varying from $\sim 100 \mathrm{kpc}$ in the cluster center to $\sim 400 \mathrm{kpc}$ in the outskirts, to the spatial distribution of the spectroscopically confirmed members. We define significant values of $-\log P_{K S}\left(D>D_{o b s}\right)$ as those which occur only $5 \%$ of the time in the 10,000 Monte Carlo realizations, and these are highlighted by the bold bubbles in Figure 13 Visual inspection of the distribution of large clustered bubbles in Figure 13 confirms the significance of the measured $\kappa_{n}$, as there appear to be four conglomerations of significant bubbles, each coincident with an increase in projected galaxy density.

Having verified the existence of substructure within Abell 1201, we further utilize the spatial and velocity information of our cluster galaxies by allocating group membership using the Kaye's Mixture Model (KMM) al- gorithm of Ashman et al. (1994). The algorithm fits a user specified number of $\mathrm{N}$-dimensional Gaussians to the data and determines the improvement of the fit over that of a single N-dimensional Gaussian via a maximum likelihood test. The major drawbacks of this method are that the spatial distribution of the galaxies does not follow a Gaussian shape (although the velocity distribution does for a relaxed cluster), and the number of Gaussians needs to be known a priori. Visual inspection of the projected $\mathrm{X}$ and $\mathrm{Y}$ galaxy distributions reveal they are at least qualitatively Gaussian, and given the benefit of including an extra two dimensions in the analysis far outweighs the false assumption of Gaussianity, we proceed with the full 3-D KMM analysis. Overcoming the latter drawback requires a robust method of estimating the initial number of Gaussians, and also their parameters. Given the correlation between the significant bubbles and galaxy surface density peaks seen in Figure 13, we use it as a guide to estimate the positions and projected radii of substructures. There appear to be 6 spatially separated substructures where the local velocity distribution differs significantly from the global one, along with the main Abell 1201 cluster. We inspect the velocity distributions of all galaxies within the estimated projected radius for each substructure, excise any obvious interlopers and determine the median and standard deviation of the $\mathrm{X}$ position $(\mathrm{kpc})$, $\mathrm{Y}$ position $(\mathrm{kpc})$ and velocity distributions for the remaining substructure galaxies. These parameters serve as initial estimates for input into the KMM algorithm, and are presented in table 4, along with the outputs from the KMM algorithm, where $(\bar{x}, \bar{y}, \bar{v})$ are the means of the distributions, $\left(\sigma_{x}, \sigma_{y}, \sigma_{v}\right)$ the standard deviations, $N_{g a l}$ is the number of galaxies in the substructure and Rate is the estimate of the overall rate for correct allocation of galaxies to this substructure.

Given that the substructures KMM3 and KMM4 are close both spatially and in velocity, as are KMM5 and KMM6, it is possible that they are part of the same structures. We therefore combined the inputs for the four substructures into $\mathrm{KMM}(3+4)$ and $\mathrm{KMM}(5+6)$ and re-ran the KMM algorithm on the 5 substructures. The results for KMM1, KMM2 and KMM7 were very similar to those found using 7 partitions, with similar galaxies being allocated to the combined $\operatorname{KMM}(3+4)$ and $\operatorname{KMM}(5+6)$ systems as were allocated when considering them as separate entities, and the overall correct allocation estimator for both the 5 and 7 substructure analyses being $98 \%$ and $97.9 \%$, respectively. It appears the algorithm does not favor a 7 structure partition over a 5 structure partition, so we used the simpler decomposition and assumed the 5 substructure partition is the correct one. We tested the assumption of Gaussianity for the velocity distribution of each substructure using a K-S test and found no evidence for significant deviations from Gaussianity. The results of the allocations to the different substructures are plotted in the top panel of Figure 14, where we have color-coded the symbols to match the respective velocity distributions plotted in the bottom panel of Figure 14.

\section{MERGER SCENARIO}

Both the X-ray and optical analyses give clear indications that Abell 1201 hosts multiple substructures. In this section we first give a qualitative scenario explaining the appearance of the X-ray structure and cold 
TABLE 4

Results of the KMM analysis for partitioning the data into 7 (top) and 5 (bottom) substructures. See Figure 14 for the SPATIAL AND VELOCity Distributions of THE 5 PARTition RESUlts.

\begin{tabular}{|c|c|c|c|c|c|c|c|}
\hline \multirow[b]{2}{*}{ Group } & \multicolumn{3}{|c|}{ Initial Inputs } & \multicolumn{4}{|c|}{ KMM Outputs } \\
\hline & $(\bar{x}, \bar{y}, \bar{v})$ & $\left(\sigma_{x}, \sigma_{y}, \sigma_{v}\right)$ & $N_{\text {gal }}$ & $(\bar{x}, \bar{y}, \bar{v})$ & $\left(\sigma_{x}, \sigma_{y}, \sigma_{v}\right)$ & $N_{\text {gal }}$ & Rate $(\%)$ \\
\hline \multicolumn{8}{|c|}{7 substructures } \\
\hline KMM1 & $(138,388,444)$ & $(103,64,175)$ & 9 & $(150,383,432)$ & $(145,121,167)$ & 12 & 100 \\
\hline KMM2 & $(-1125,550,-1816)$ & $(320,283,318)$ & 11 & $(-890,346,-1887)$ & $(419,224,254)$ & 14 & 100 \\
\hline KMM3 & $(-431,-444,-996)$ & $(62,134,221)$ & 7 & $(-462,-427,-1008)$ & $(44,139,160)$ & 6 & 100 \\
\hline KMM4 & $(-869,-63,-607)$ & $(134,116,383)$ & 12 & $(-924,-58,-672)$ & $(146,120,257)$ & 12 & 100 \\
\hline KMM5 & $(1563,513,-642)$ & $(200,139,233)$ & 11 & $(1650,533,-769)$ & $(180,132,208)$ & 10 & 98 \\
\hline KMM6 & $(2150,1213,-236)$ & $(155,249,170)$ & 9 & $(2037,1429,-317)$ & $(401,459,220)$ & 15 & 96 \\
\hline KMM7 & $(0,0,2)$ & $(1236,979,738)$ & 262 & $(-96,93,133)$ & $(1211,1016,682)$ & 252 & 98 \\
\hline \multicolumn{8}{|c|}{5 substructures } \\
\hline KMM1 & $(138388,444)$ & $(103,64,175)$ & 9 & $(151,382,432)$ & $(145,121,166)$ & 12 & 100 \\
\hline KMM2 & $(-1125,550,-1816)$ & $(320,283,318)$ & 11 & $(-890,346,-1886)$ & $(419,224,255)$ & 14 & 100 \\
\hline $\operatorname{KMM}(3+4)$ & $(-720,-200,-777)$ & $(239,223,321)$ & 19 & $(-684,-249,-867)$ & $(304,264,211))$ & 16 & 94 \\
\hline $\operatorname{KMM}(5+6)$ & $(1884,849,-608)$ & $(323,421,246)$ & 20 & $(1831,900,-753)$ & $(348,452,328)$ & 17 & 99 \\
\hline KMM7 & $(0,0,2)$ & $(1236,979,738)$ & 262 & $(-32,138,127)$ & $(1250,1028,663)$ & 262 & 98 \\
\hline
\end{tabular}

Note. - The units of $\bar{x}, \bar{y}, \sigma_{x}$ and $\sigma_{y}$ are kpc, and the units of $\bar{v}$ and $\sigma_{v}$ are $\mathrm{km} \mathrm{s}^{-1}$.

fronts through simple interpretations of the optical and X-ray observations combined with hydrodynamic simulations of Poole et al. (2006) and Ascasibar \& Markevitch (2006). Second we use two-body analytic models to determine which of the substructures are bound to the main cluster.

\subsection{KMM1, the north-west X-ray excess and the formation of the cold fronts}

Figure 2 shows an SDSS $r$-band image of the central regions of Abell 1201 with X-ray contours overlaid, along with regions showing the cluster members and KMM1 allocations. Clearly, KMM1 is coincident with the excess X-ray emission which is probably the remnant of the gas core of KMM1. The morphology of the X-ray emission indicates the remnant KMM1 core is breaking up, and there appears to be a tail pointing towards the main cluster, although it is difficult to disentangle the cluster and subclump emission. The positioning of the cold fronts on opposite sides of the cluster center, along the direction to the north-west clump, suggests motion of the cluster core in this direction. This evidence points to a scenario where KMM1 has made its closest approach to the core of Abell 1201 and is traveling outwards toward the north-west. Projection effects make it difficult to know on exactly which plane the merger is occurring, although the small radial velocity offset between KMM1 and Abell 1201 and the location of the cold fronts suggest the majority of the subcluster motion is in the plane of the sky. Since the core of Abell 1201 is not completely disrupted and is coincident with the dominant cluster galaxy, which presumably lies at the cluster potential minimum, it is unlikely KMM1 passed directly through the cluster core (Poole et al. 2008). The low velocity dispersion and compact galaxy distribution of KMM1 suggest we are seeing the surviving central region of a once larger cluster which has been stripped of its outer members due to the tidal effects of the main Abell 1201 cluster potential.

The south-east cold front appears to be connected to the core of the main cluster, as evidenced by the signifi- cant residuals extending from the core to the cold front, and also by the finger of cold $(\sim 4 \mathrm{keV})$ gas joining the core and the cold front seen in the temperature map. This suggests the gas causing the cold front was once part of the core and has been displaced by the merger. It is possible that the cold fronts we see are the result of spirals of intertwined low and high entropy gas, as seen in both simulations Ascasibar \& Markevitch 2006; Poole et al. 2006) and observations (Fabian et al. 2006; Clarke et al. 2004), with the rotation axis of the spirals roughly in the plane of the sky. This scenario is capable of producing several features observed in Abell 1201: two cold fronts at different distances on opposite sides of the core, the coincidence of the X-ray core with the dominant cluster galaxy and the region of hot gas on the north-west side of the cluster core which was probably heated to its observed temperature by compression or shock heating caused by the merger with KMM1. To illustrate this we present Figure 15 which shows three temperature maps from the offset $\left(r_{\min }=360 \mathrm{kpc}\right), 3$ to 1 mass ratio merger simulation of Poole et al. (2006) generated using emission weighted temperatures integrated along a $3.5 \mathrm{Mpc}$ line-of-sight. There are 3 projections each at 0.7 Gyrs after pericentric passage with one projection viewing along an axis perpendicular to the plane of the merger orbit $(x-y)$ and two projections orthogonal to this. This makes apparent the effect of projection - the spiral type structure produces cold fronts observable over a wide range of viewing angles. Qualitatively, the map shown in the middle panel of Figure 15 reproduces the features of Abell 1201 noted above, although the secondary core appears farther from the cluster center than that in Abell 1201. If the orbit of the secondary core is close to our line-of-sight and the core is close to turn around, this might be resolved by projecton effects. However, the simulation illustrated has generic initial conditions, not specific to Abell 1201. In particular, it is possible that a closer core passage could produce the structure observed in Abell 1201 without appealing to fortuitous viewing conditions. The fact that an ideal- 

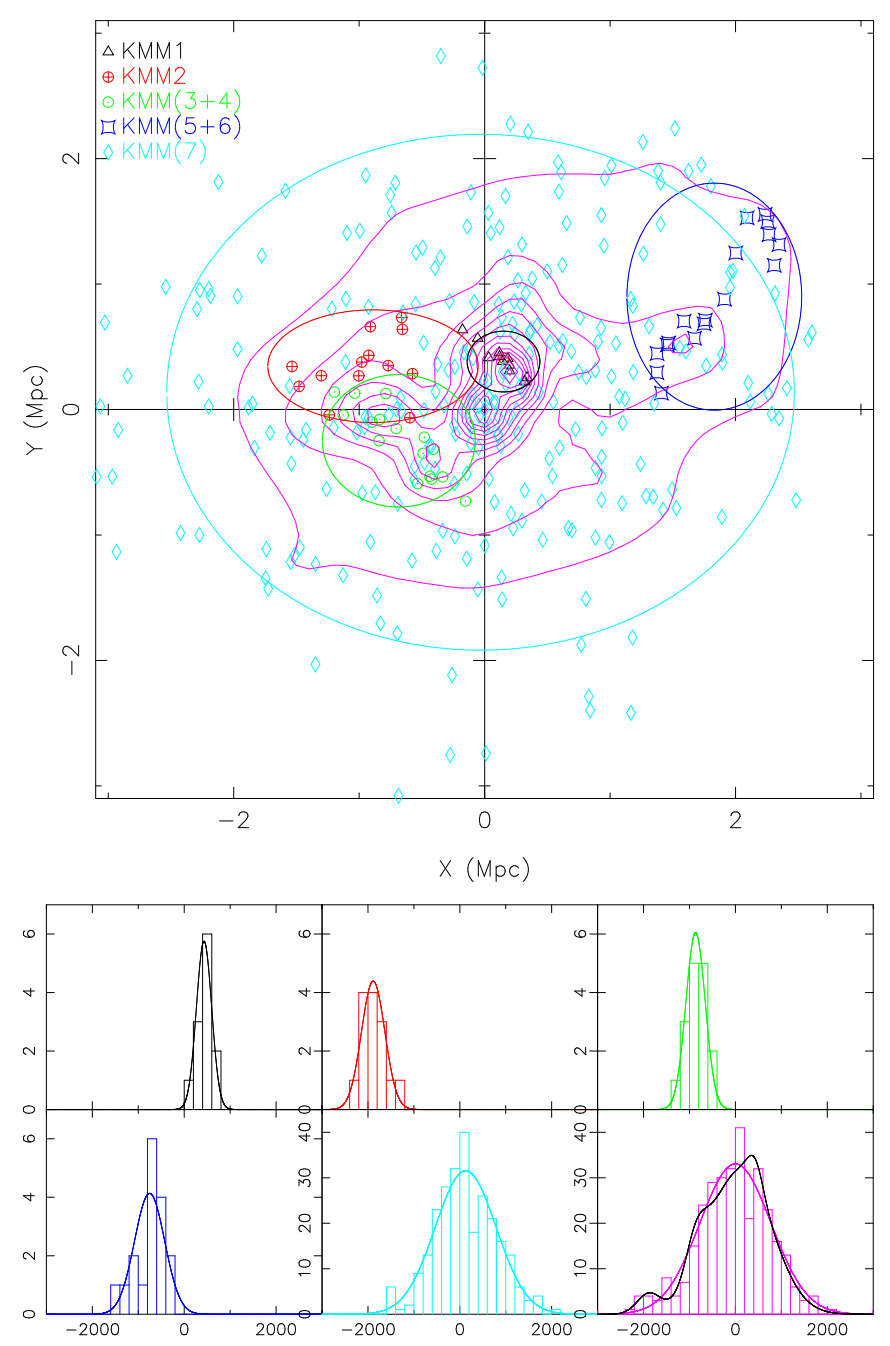

FIG. 14.- Top Panel: The spatial distribution of the different partitions assigned by the KMM analysis. The ellipses show the $2 \sigma$ contours for the Gaussians fitted to the respective spatial distributions. We also plot the galaxy surface density contours in pink (same spacing as Fig. 13). Bottom panel: The velocity distributions of the different partitions; the curved lines represent Gaussian functions whose mean and $\sigma$ are equal to the KMM $\bar{v}$ and $\sigma_{v}$ values. The color coding in the bottom panel matches the key in the top left of the top panel. The bottom right-most velocity distribution shows the whole cluster sample with a Gaussian generated using the biweight estimators overplotted in pink; the combination of all the KMM Gaussians is plotted in black.

ized simulation reproduces the majority of the observed features supports the proposed merger scenario. Future simulations specific to Abell 1201 will determine which of these possibilities can better explain its observed features.

It appears Abell 1201 is an excellent example of a "sloshing" type cold front cluster, where the perturber is still clearly visible in the X-ray and optical observations (in the form of KMM1), similar to Abell 1644 (see Figure 17 Markevitch \& Vikhlinin 2007). Thus, Abell 1201 is an excellent candidate for follow up detailed simulations in order to derive an accurate picture of exactly how the system has evolved to its current state, and whether it will evolve into a relaxed looking cluster harboring cold fronts with no discernable perturber (eg., RXJ1720.1+2638, MS1455.0+2232 or Abell 2029 Markevitch \& Vikhlinin 2007; Mazzotta \& Giacintucci 2008)

\subsection{Two-body merger dynamics}

In this section we apply the two-body dynamical analysis first implemented by Beers et al. (1982) to the substructures detected in 33.3 .3 with the aim of determining which of the substructures are bound to the main cluster, and to give an initial idea of the internal dynamics of the cluster. The model allows estimation of the probability that a substructure is unbound and lying close to the main cluster along the line of sight, and also allows the calculation of the probability that a substructure is bound and collapsing or bound and expanding.

The accuracy of the results given by the model rely critically on the following assumptions: the orbits are radial, the cluster masses are concentrated into a point at the respective centers, the clusters had zero initial separation at $\mathrm{t}=0$, and they are moving apart or coming together for the first time. These assumptions are extremely unlikely to hold true for KMM1, which probably resides well within the cluster virial radius, deep within the main cluster potential well (where dynamical friction, tidal forces and angular momentum all become significant) and is apparently currently heading to the north-west after pericentric passage. Thus, we do not present results from the two-body analysis for KMM1, and defer quantitative analysis of the KMM1 merger until detailed simulations are available, noting only that given the observations presented here, it appears KMM1 and Abell 1201 form a bound system.

As inputs, the model requires information about the projected spatial separation, $R_{p}$, the line of sight velocity difference, $V_{r}$, and the total mass of the system, returning possible solutions for $\alpha$ (the angle between the line joining the two clusters and the line of sight), the total mass required to bind the system, and the true 3 -D spatial separation, $R$, and velocity difference, $V$. The parametric solutions to the equations of motion for bound radial orbits are

$$
\begin{gathered}
V=\frac{V_{R}}{\sin \alpha}=\left(\frac{2 G M}{R_{m}}\right)^{1 / 2} \frac{\sin \chi}{(1-\cos \chi)}, \\
t=\left(\frac{R_{m}^{3}}{8 G M}\right)^{1 / 2}(\chi-\sin \chi), \\
R=\frac{R_{p}}{\cos \alpha}=\frac{R_{m}}{2}(1-\cos \chi),
\end{gathered}
$$

where $R_{m}$ is the maximum separation of the two clusters at turn around, $M$ is the total mass of the system, and $\chi$ is the developmental angle which varies from $0<\chi<2 \pi$ with $\chi=0,2 \pi$ being the stages of the orbit corresponding to zero spatial separation. It is also possible to solve for the unbound case, where the parametric solutions are

$$
\begin{gathered}
V=\frac{V_{r}}{\sin \alpha}=V_{\infty} \frac{\sinh \chi}{(\cosh \chi-1)}, \\
t=\frac{G M}{V_{\infty}^{3}}(\sinh \chi-\chi), \\
R=\frac{R_{p}}{\cos \alpha}=\frac{G M}{V_{\infty}^{2}}(\cosh \chi-1),
\end{gathered}
$$

where $V_{\infty}$ is the asymptotic expansion velocity. 

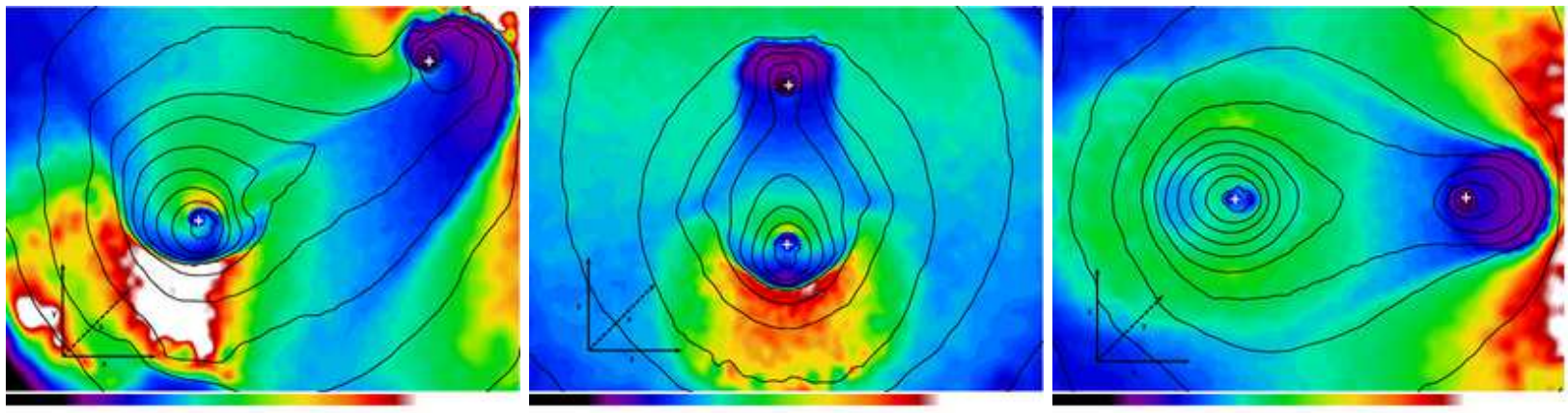

FIG. 15. - Snapshot temperature maps of the 3 to 1 mass ratio, offset $\left(r_{\min }=360 \mathrm{kpc}\right)$ merger simulation from Poole et al. (2006) taken 0.7 Gyrs after pericentric passage. The left panel shows the merger viewed from a vantage point where the line of sight is perpendicular to the merger orbital plane, while for the middle panel the line of sight is along the $\mathrm{x}$-axis and the right panel the line of sight is along the y-axis. The vectors on the lower left of each panel show this, and the length of each vector has physical size $500 \mathrm{kpc}$. The color scale runs from black to white with black showing the lowest temeperatures, and white the highest (on an arbitrary temperature scale). X-ray surface brightness contours are overlaid and the white crosses show the positions of the peak in the dark matter density for the primary and secondary clusters.

For the bound case, combining equations 8,9 and 10 gives

$$
\tan \alpha=\frac{\mathrm{V}_{\mathrm{r}} \mathrm{t}}{\mathrm{R}_{\mathrm{p}}} \frac{(1-\cos \chi)^{2}}{\sin \chi(\chi-\sin \chi)},
$$

and, similarly, we obtain

$$
\tan \alpha=\frac{\mathrm{V}_{\mathrm{r}} \mathrm{t}}{\mathrm{R}_{\mathrm{p}}} \frac{(\cosh \chi-1)^{2}}{\sinh \chi(\sinh \chi-\chi)} .
$$

for the unbound case. Assuming $t=11.38$ Gyrs, i.e. the age of the Universe at $z=0.168$ in our assumed cosmology, for values of the parameter $\chi$ in the range 0 to $2 \pi$ these equations determine possible solutions for $\alpha$. Given that the mass of the system is the least well constrained of the input parameters, we solved for $M$ as a function of $\alpha$ using as input $V_{r}$ and $R_{p}$ for each group in the KMM analysis. Here $R_{p}$ was calculated as the distance from the KMM centroid to the central dominant galaxy, rather than the KMM7 center, which is slightly offset from the central dominant galaxy position. $V_{r}$ is the radial velocity offset of the KMM partition of interest, taken with respect to the biweight location of peculiar velocities in KMM7, where either the median (for KMM groups with $\left.N_{\text {gal }}<15\right)$ or the biweight locator $\left(N_{\text {gal }}>15\right)$ was used to determine the group's peculiar velocity. We plot the possible solutions of $M$ as a function of $\alpha$ in Figure 16.

Determination of solutions for the orbits of the systems requires knowledge of the total mass of the system. Given the velocity dispersions of the substructures in Abell 1201 are much smaller than that of the main cluster, it is reasonable to assume that when compared to the mass of the main Abell 1201 cluster the contribution of the masses of the subclumps is negligible. Hence we assumed the total system mass was equivalent to the virial mass of Abell 1201. We used the methodology of Girardi et al. (1998) for the determination of the mass

$$
M=M_{v i r}-C=\frac{3 \pi}{2} \frac{\sigma_{v}^{2} R_{P V}}{G}-C
$$

where $C$ is the surface term correction accounting for the lack of coverage for the entire cluster (i.e. out to the turn around radius). Here we took $C=0.19 M_{v i r}$, which is the median value derived in Girardi et al. (1998), and $\sigma_{v}=$ $665 \pm 32 \mathrm{~km} \mathrm{~s}^{-1}$ which is the biweight scale estimator derived for galaxies assigned to KMM7 and

$$
R_{P V}=\frac{N_{v i r}\left(N_{v i r}-1\right)}{\sum_{i=j+1}^{N_{v i r}} \sum_{j=1}^{i-1} R_{i j}^{-1}},
$$

which was determined within $r_{200}=0.17 \sigma_{v} / H(z)=$ $1.5 \mathrm{Mpc}$ (Carlberg et al. 1997) where $N_{v i r}=154$ is the number of KMM7 members within $r_{200}$, and $R_{i j}$ is the projected distance between two galaxies. We determined $R_{P V}=1.5 \pm 0.1 \mathrm{Mpc}$ and $M=5.9( \pm 0.6) \times 10^{14} \mathrm{M}_{\odot}$ where the error in $R_{P V}$ was determined using the Jackknife technique, and standard error propagation was used to derive the error in the mass.

The possible solutions for the orbits are given by the intersection of the line representing the Abell 1201 virial mass estimate with the curve for $M$ as a function of $\alpha$ in Figure 16. For each solution, we present the associated probability relative to the other solutions by considering the range of possible $\alpha$ given by the upper and lower values, $\alpha_{U}$ and $\alpha_{L}$, respectively, derived from the error bounds on each curve, and assuming that each individual solution is equally probable. The relative probability is

$$
p_{\text {rel }}=\frac{\left(\int_{\alpha_{L}}^{\alpha_{U}} \cos \alpha \mathrm{d} \alpha\right)}{\sum p_{\text {rel }}},
$$

for the solution of interest, and

$$
\sum p_{r e l}=p_{B O}+p_{U O}+p_{B I_{a}}+p_{B I_{b}}
$$

where $p_{B O}, p_{U O}, p_{B I_{a}}, p_{B I_{b}}$ are the $\int_{\alpha_{L}}^{\alpha_{U}} \cos \alpha \mathrm{d} \alpha$ values for the bound outgoing, unbound outgoing, first bound incoming and second bound incoming solutions, respectively (Brough et al. 2006). We present the results in Table 5 .

We also determined the probability the subclumps are bound using the Newtonian binding criterion, where a two-body system is bound if the kinetic energy is less than or equal to the potential energy. The criterion is given by Beers et al. (1982) as

$$
V_{r}^{2} R_{p} \leq 2 G M \sin ^{2} \alpha \cos \alpha .
$$

This curve is also plotted in Figure 16, and the probability is $P_{\text {bound }}=\int_{\alpha_{L}}^{\alpha_{U}} \cos \alpha \mathrm{d} \alpha$, where $\alpha_{L}$ and $\alpha_{U}$ are determined from the intersections of $M_{v i r}$ and the curve 


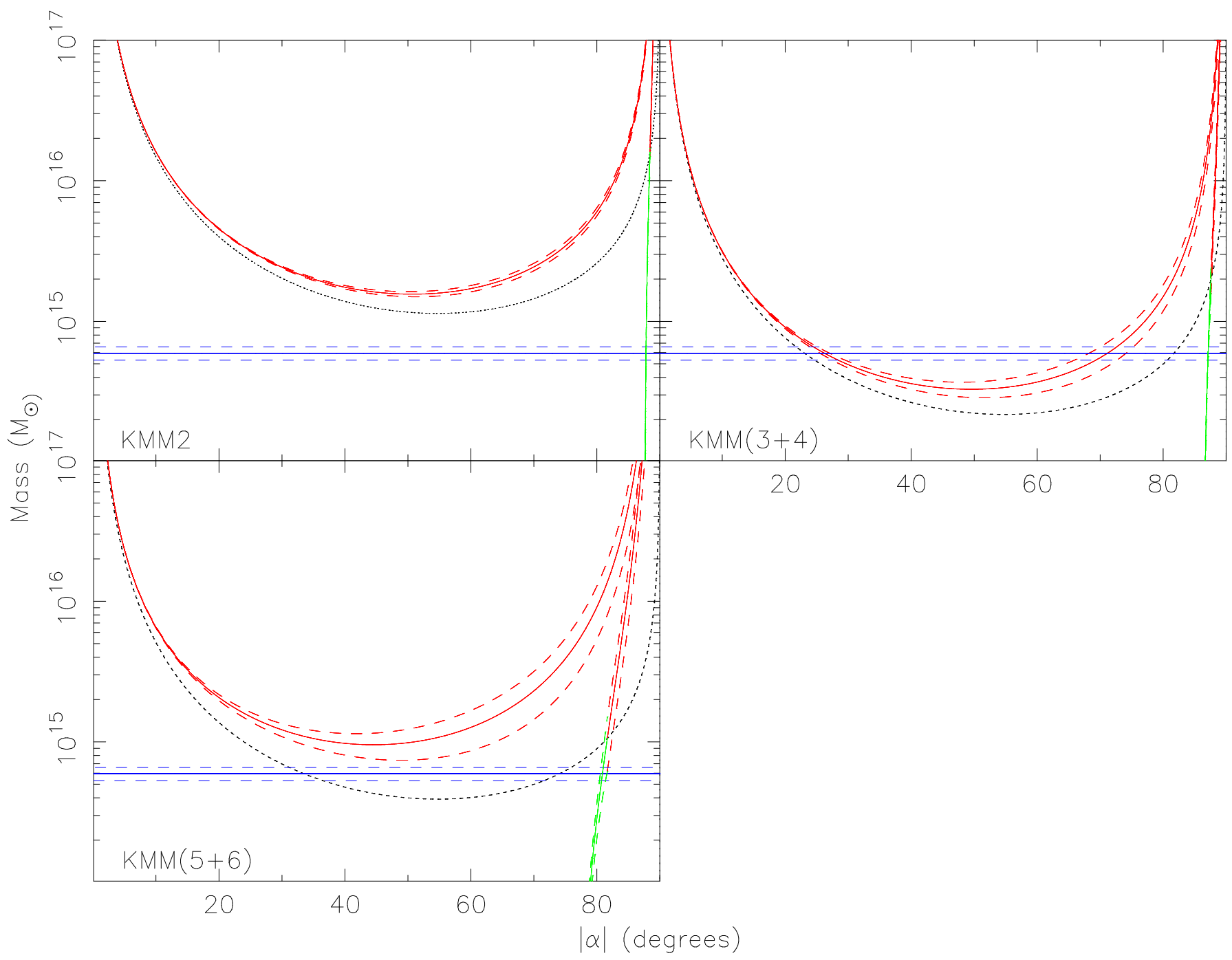

Fig. 16.- Binding mass as a function of $\alpha$ (orange and green curves). The orange curves correspond to bound solutions, while the green curves represent unbound solutions. The dashed curves show the $1 \sigma$ errors. The blue line shows the mass derived using galaxies allocated to KMM7 and the dashed blue lines indicate the upper and lower $1 \sigma$ errors. The small black dashed line delineates the bound and unbound regions according to the Newtonian binding criterion.

TABLE 5

Probabilities that the detected substructures are bound to the Abell 1201 Cluster, DERIVEd USing the two-body dynamical analysis and the NeWtonian Criterion (See text).

\begin{tabular}{cccccccc}
\hline \hline Subclump & $\begin{array}{c}V_{r} \\
\left(\mathrm{~km} \mathrm{~s}^{-1}\right)\end{array}$ & $\begin{array}{c}R_{P} \\
(\mathrm{Mpc})\end{array}$ & $\begin{array}{c}P_{B I_{a}} \\
(\text { per cent) }\end{array}$ & $\begin{array}{c}P_{B I_{b}} \\
(\text { per cent) }\end{array}$ & $\begin{array}{c}P_{B O} \\
(\text { per cent) }\end{array}$ & $\begin{array}{c}P_{U O} \\
\text { (per cent) }\end{array}$ & $\begin{array}{c}P_{\text {bound }} \\
\text { (per cent) }\end{array}$ \\
\hline KMM2 & $-1989 \pm 46$ & 0.95 & - & - & - & 100 & - \\
KMM(3+4) & $-996 \pm 72$ & 0.73 & 62 & 38 & - & $<<1$ & 60 \\
KMM(5+6) & $-798 \pm 113$ & 2.04 & - & - & $<<1$ & 100 & 41 \\
\hline
\end{tabular}

representing the Newtonian criterion. We present the results in Table 5 .

Considering the Newtonian criterion and two-body solutions, we find that there is a high probability of $\operatorname{KMM}(3+4)$ being bound to Abell 1201, with two solutions which are, roughly speaking, as probable as each other. The most probable solution is $\mathrm{KMM}(3+4)$ is bound and incoming with $\alpha=26$ degrees, $R=0.8 \mathrm{Mpc}$ and $V=-2252 \mathrm{~km} \mathrm{~s}^{-1}$, whilst the slightly less probable solution is also bound and incoming with $\alpha=71$ degrees,
$R=2.3 \mathrm{Mpc}$ and $V=-1052 \mathrm{kms}^{-1}$. If KMM1 is outbound to the north-west, as proposed in $\S$ 4.1, then it is possible that $\operatorname{KMM}(3+4)$ consists of tidal debris stripped from KMM1 during is approach to pericentric passage from the south-east. Further evidence for a tidal origin comes from the spatial distribution of galaxies, where $\mathrm{KMM}(3+4)$ appears as two conglomerations in Figure 16, although simulations are required to confirm this.

In the case of KMM2, we find only unbound out- 
going solutions with the most probable solution giving $\alpha=-88$ degrees (i.e. it's motion is aligned at $2 \mathrm{deg}$ to the line of sight), $R=24.8 \mathrm{Mpc}$ and $V=1990 \mathrm{~km} \mathrm{~s}^{-1}$. Thus it is likely that KMM2 is a group of galaxies lying in the foreground moving away from Abell 1201, and not physically associated. $\mathrm{KMM}(5+6)$ has a best fitting unbound outgoing solution, although within the errors this may be bound outgoing, with $\alpha=-81$ degrees, $R=12.9 \mathrm{Mpc}$ and $V=808 \mathrm{~km} \mathrm{~s}^{-1}$. Given the Newtonian criterion allows $\operatorname{KMM}(5+6)$ to be bound and that it is possible we have underestimated the mass within the radius at which $\mathrm{KMM}(5+6)$ lies, it is entirely plausible that we have also underestimated the binding probability for $\operatorname{KMM}(5+6)$.

The two body analysis presented here has allowed the determination of which substructures are most likely bound to the main cluster. This gives an initial understanding of which substructures are important in understanding the internal dynamics of Abell 1201, which will be further modeled in future simulations and presented in a forthcoming paper.

\section{SUMMARY AND CONCLUSIONS}

We have presented an analysis of Abell 1201 using both Chandra X-ray data and optical spectroscopic data obtained using the AAT/AAOmega and MMT/Hectospec MOS. The X-ray analysis reveals the following:

1. An elliptical morphology with two surface brightness discontinuities positioned roughly on an axis joining the main cluster with a clump of excess emission to the north-west. The density, temperature and pressure jumps across these discontinuities are consistent with cold fronts.

2. A residual map obtained by subtracting a double beta model from the surface brightness distribution shows significant residuals at the position of the north-west clump. It shows significant residuals extending from the cluster core to the south-east cold front.

3. A temperature map shows that south-east residual is coincident with a finger of cold gas which also extends from the cluster core and terminates on the inner side of the front.

4. There is also a significantly hotter region on the north-west side of the inner cold front between the main cluster core and the subclump further to the north-west.

The optical MOS analysis reveal:

1. From 321 cluster member spectra the cluster redshift is $\mathrm{z}=0.1673$ and the velocity dispersion is $778 \mathrm{~km} \mathrm{~s}^{-1}$.

2. The velocity distribution is not significantly nonGaussian, despite the clearly disturbed nature of Abell 1201 evidenced by the X-ray data.

3. Combining the peculiar velocity and spatial information reveals significant localized velocity substructure.
4. Using the KMM method of Ashman et al. (1994) the substructure can be partitioned into 5 distinct clumps - the main Abell 1201 cluster with 262 members (KMM7), an infalling subgroup $410 \mathrm{kpc}$ to the north-west coincident with the Xray excess containing 12 members (KMM1), a subgroup $\sim 950 \mathrm{kpc}$ to the NE with 14 members (KMM2), a subgroup $\sim 730 \mathrm{kpc}$ to the south-east with 16 members $(\mathrm{KMM} 3+4)$ and a group $\sim 2 \mathrm{Mpc}$ to the north-west with 15 members (KMM5+6).

5. Application of the two-body dynamical analysis of Beers et al. (1982) reveals KMM2 is likely an unbound foreground group, whilst it is also possible $\operatorname{KMM}(5+6)$ is unbound and $\operatorname{KMM}(3+4)$ has a high probability of being bound to the main cluster.

The analysis presented here supports the view that the cold fronts in Abell 1201 are a direct result of merger activity. Our X-ray and optical analysis combined with the simulation of Poole et al. (2006) indicate that the KMM1 subcluster has come from the south-east and is currently heading towards the north-west after an offset passage past the main cluster core. Disturbance caused by the passage of this subcluster has set off sloshing of the cool gas in the core of the main cluster, which produced two concentric cold fronts around the main core. The value of the use of multiple methods of merger detection for analyzing a clusters dynamical state is also confirmed in this study.

The next step in our study of cold front clusters will be to utilize radio data in combination with the optical spectroscopy presented here to study the effects of the merger on the galaxy population, and search for signs of non-thermal radio halo/relic emission. Deeper X-ray observations will help place tighter constraints on the parameters derived here, whilst allowing a more detailed analysis of the complex X-ray structure.

\section{ACKNOWLEDGMENTS}

We thank David Woods for useful discussions and sanity checks. We are grateful to Will Saunders and the staff at the Anglo-Australian Observatory for their support during AAT observations. Observations reported here were obtained at the MMT Observatory, a joint facility of the Smithsonian Institution and the University of Arizona. We thank the MMT operators and queueschedule mode scientists for their help during observations and the staff at the Harvard-Smithsonian Center for Astrophysics Telescope Data Center for reducing the Hectospec data.

This research has made use of software provided by the Chandra X-ray Center (CXC) in the application packages CIAO, ChIPS, and Sherpa and also of data obtained from the Chandra archive at the NASA Chandra X-ray center (http://cxc.harvard.edu/cda/). This research has made use of the NASA/IPAC Extragalactic Database (NED) which is operated by the Jet Propulsion Laboratory, California Institute of Technology, under contract with the National Aeronautics and Space Administration.

MSO was supported by an Australian Postgraduate Award, and acknowledges the hospitality of the HarvardSmithsonian Center for Astrophysics where a portion of this study was undertaken. We acknowledge the financial support of the Australian Research Council (via 
its Discovery Project Scheme) throughout the course of

01130.

this work. PEJN was supported by NASA grant NAS8-

\section{REFERENCES}

Abell, G. O., Corwin, Jr., H. G., \& Olowin, R. P. 1989, ApJS, 70, 1

Arnaud, K. A. 1996, in Astronomical Society of the Pacific Conference Series, Vol. 101, Astronomical Data Analysis Software and Systems V, ed. G. H. Jacoby \& J. Barnes, 17-+ Ascasibar, Y., \& Markevitch, M. 2006, ApJ, 650, 102

Ashman, K. M., Bird, C. M., \& Zepf, S. E. 1994, AJ, 108, 2348

Barrena, R., Boschin, W., Girardi, M., \& Spolaor, M. 2007, A\&A, 467, 37

Beers, T. C., Flynn, K., \& Gebhardt, K. 1990, AJ, 100, 32

Beers, T. C., Geller, M. J., \& Huchra, J. P. 1982, ApJ, 257, 23

Böhringer, H., Voges, W., Huchra, J. P., McLean, B., Giacconi, R., Rosati, P., Burg, R., Mader, J., Schuecker, P., Simiç, D., Komossa, S., Reiprich, T. H., Retzlaff, J., \& Trümper, J. 2000, ApJS, 129, 435

Boschin, W., Girardi, M., Barrena, R., Biviano, A., Feretti, L., \& Ramella, M. 2004, A\&A, 416, 839

Brough, S., Forbes, D. A., Kilborn, V. A., Couch, W., \& Colless, M. 2006, MNRAS, 369, 1351

Carlberg, R. G., Yee, H. K. C., Ellingson, E., Morris, S. L., Abraham, R., Gravel, P., Pritchet, C. J., Smecker-Hane, T., Hartwick, F. D. A., Hesser, J. E., Hutchings, J. B., \& Oke, J. B. 1997, ApJ, 485, L13+

Carrasco, E. R., Cypriano, E. S., Neto, G. B. L., Cuevas, H., Sodré, Jr., L., de Oliveira, C. M., \& Ramirez, A. 2007, ApJ, 664, 777

Churazov, E., Forman, W., Jones, C., \& Böhringer, H. 2003, ApJ, 590,225

Clarke, T. E., Blanton, E. L., \& Sarazin, C. L. 2004, ApJ, 616, 178 Colless, M., Dalton, G., Maddox, S., Sutherland, W., Norberg, P., Cole, S., Bland-Hawthorn, J., Bridges, T., Cannon, R., Collins, C., Couch, W., Cross, N., Deeley, K., De Propris, R., Driver, S. P., Efstathiou, G., Ellis, R. S., Frenk, C. S., Glazebrook, K. Jackson, C., Lahav, O., Lewis, I., Lumsden, S., Madgwick, D., Peacock, J. A., Peterson, B. A., Price, I., Seaborne, M., \& Taylor, K. 2001, MNRAS, 328, 1039

Colless, M., \& Dunn, A. M. 1996, ApJ, 458, 435

Cross, N. J. G., Driver, S. P., Liske, J., Lemon, D. J., Peacock, J. A., Cole, S., Norberg, P., \& Sutherland, W. J. 2004, MNRAS, 349, 576

De Propris, R., Couch, W. J., Colless, M., Dalton, G. B., Collins, C., Baugh, C. M., Bland-Hawthorn, J., Bridges, T., Cannon, R., Cole, S., Cross, N., Deeley, K., Driver, S. P., Efstathiou, G. Ellis, R. S., Frenk, C. S., Glazebrook, K., Jackson, C., Lahav, O., Lewis, I., Lumsden, S., Maddox, S., Madgwick, D., Moody, S., Norberg, P., Peacock, J. A., Percival, W., Peterson, B. A., Sutherland, W., \& Taylor, K. 2002, MNRAS, 329, 87

den Hartog, R., \& Katgert, P. 1996, MNRAS, 279, 349

Dickey, J. M., \& Lockman, F. J. 1990, ARA\&A, 28, 215

Dressler, A., \& Shectman, S. A. 1988, AJ, 95, 985

Fabian, A. C., Sanders, J. S., Taylor, G. B., Allen, S. W., Crawford, C. S., Johnstone, R. M., \& Iwasawa, K. 2006, MNRAS, 366, 417

Fabricant, D., Fata, R., Roll, J., Hertz, E., Caldwell, N., Gauron, T., Geary, J., McLeod, B., Szentgyorgyi, A., Zajac, J., Kurtz, M., Barberis, J., Bergner, H., Brown, W., Conroy, M., Eng, R., Geller, M., Goddard, R., Honsa, M., Mueller, M., Mink, D., Ordway, M., Tokarz, S., Woods, D., Wyatt, W., Epps, H., \& Dell'Antonio, I. 2005, PASP, 117, 1411

Fadda, D., Girardi, M., Giuricin, G., Mardirossian, F., \& Mezzetti, M. 1996, ApJ, 473, 670

Fujita, Y., Sarazin, C. L., Reiprich, T. H., Andernach, H., Ehle, M., Murgia, M., Rudnick, L., \& Slee, O. B. 2004, ApJ, 616, 157 Geller, M. J., \& Beers, T. C. 1982, PASP, 94, 421

Giovannini, G., \& Feretti, L. 2002, in Astrophysics and Space Science Library, Vol. 272, Merging Processes in Galaxy Clusters, ed. L. Feretti, I. M. Gioia, \& G. Giovannini, 197-227

Girardi, M., \& Biviano, A. 2002, in Astrophysics and Space Science Library, Vol. 272, Merging Processes in Galaxy Clusters, ed. L. Feretti, I. M. Gioia, \& G. Giovannini, 39-77

Girardi, M., Boschin, W., \& Barrena, R. 2006, A\&A, 455, 45

Girardi, M., Giuricin, G., Mardirossian, F., Mezzetti, M., \& Boschin, W. 1998, ApJ, 505, 74

Johnston-Hollitt, M., Hunstead, R. W., \& Corbett, E. 2008, A\&A, 479,1
Kaastra, J. S. 1992, An X-Ray Spectral Code for Optically Thin Plasmas (Internal SRON-Leiden Report, updated version 2.0)

Kurtz, M. J., Mink, D. J., Wyatt, W. F., Fabricant, D. G., Torres, G., Kriss, G. A., \& Tonry, J. L. 1992, in Astronomical Society of the Pacific Conference Series, Vol. 25, Astronomical Data Analysis Software and Systems I, ed. D. M. Worrall, C. Biemesderfer, \& J. Barnes, 432-+

Liedahl, D. A., Osterheld, A. L., \& Goldstein, W. H. 1995, ApJ, 438, L115

López-Cruz, O., Barkhouse, W. A., \& Yee, H. K. C. 2004, ApJ, 614,679

Markevitch, M., Bautz, M. W., Biller, B., Butt, Y., Edgar, R., Gaetz, T., Garmire, G., Grant, C. E., Green, P., Juda, M., Plucinsky, P. P., Schwartz, D., Smith, R., Vikhlinin, A., Virani, S., Wargelin, B. J., \& Wolk, S. 2003, ApJ, 583, 70

Markevitch, M., Sarazin, C. L., \& Vikhlinin, A. 1999, ApJ, 521, 526

Markevitch, M., \& Vikhlinin, A. 2007, Phys. Rep., 443, 1

Markevitch, M., Vikhlinin, A., \& Mazzotta, P. 2001, ApJ, 562, L153

Markevitch, M. Ponman, T. J., Nulsen, P. E. J., Bautz, M. W., Burke, D. J., David, L. P., Davis, D., Donnelly, R. H., Forman, W. R., Jones, C., Kaastra, J., Kellogg, E., Kim, D.-W., Kolodziejczak, J., Mazzotta, P., Pagliaro, A., Patel, S., Van Speybroeck, L., Vikhlinin, A., Vrtilek, J., Wise, M., \& Zhao, P. 2000, ApJ, 541, 542

Maurogordato, S., Cappi, A., Ferrari, C., Benoist, C., Mars, G., Soucail, G., Arnaud, M., Pratt, G. W., Bourdin, H., \& Sauvageot, J.-L. 2008, A\&A, 481, 593

Maurogordato, S., Proust, D., Beers, T. C., Arnaud, M., Pelló, R., Cappi, A., Slezak, E., \& Kriessler, J. R. 2000, A\&A, 355, 848

Mazzotta, P., \& Giacintucci, S. 2008, ApJ, 675, L9

Mazzotta, P., Markevitch, M., Forman, W. R., Jones, C., Vikhlinin, A., \& VanSpeybroeck, L. 2001a, ArXiv Astrophysics e-prints

Mazzotta, P., Markevitch, M., Vikhlinin, A., Forman, W. R., David, L. P., \& VanSpeybroeck, L. 2001b, ApJ, 555, 205

Miller, C. J., Krughoff, K. S., Batuski, D. J., \& Hill, J. M. 2002, AJ, 124, 1918

Neumann, D. M., \& Bohringer, H. 1997, MNRAS, 289, 123

Pinkney, J., Roettiger, K., Burns, J. O., \& Bird, C. M. 1996, ApJS, 104, 1

Poole, G. B., Babul, A., McCarthy, I. G., Sanderson, A. J. R., \& Fardal, M. A. 2008, preprint (astro-ph/0804.1552)

Poole, G. B., Fardal, M. A., Babul, A., McCarthy, I. G., Quinn, T., \& Wadsley, J. 2006, MNRAS, 373, 881

Sarazin, C. L. 2002, in Astrophysics and Space Science Library, Vol. 272, Merging Processes in Galaxy Clusters, ed. L. Feretti, I. M. Gioia, \& G. Giovannini, 1-38

Saunders, W., Bridges, T., Gillingham, P., Haynes, R., Smith, G. A., Whittard, J. D., Churilov, V., Lankshear, A., Croom, S., Jones, D., \& Boshuizen, C. 2004, in Presented at the Society of Photo-Optical Instrumentation Engineers (SPIE) Conference, Vol. 5492, Ground-based Instrumentation for Astronomy. Edited by Alan F. M. Moorwood and Iye Masanori. Proceedings of the SPIE, Volume 5492, pp. 389-400 (2004)., ed. A. F. M. Moorwood \& M. Iye, 389-400

Sharp, R., Saunders, W., Smith, G., Churilov, V., Correll, D., Dawson, J., Farrel, T., Frost, G., Haynes, R., Heald, R., Lankshear, A., Mayfield, D., Waller, L., \& Whittard, D. 2006, in Presented at the Society of Photo-Optical Instrumentation Engineers (SPIE) Conference, Vol. 6269, Ground-based and Airborne Instrumentation for Astronomy. Edited by McLean, Ian S.; Iye, Masanori. Proceedings of the SPIE, Volume 6269, pp. 62690G (2006).

Smith, G. A., Saunders, W., Bridges, T., Churilov, V., Lankshear, A., Dawson, J., Correll, D., Waller, L., Haynes, R., \& Frost, G. 2004, in Presented at the Society of PhotoOptical Instrumentation Engineers (SPIE) Conference, Vol. 5492, Ground-based Instrumentation for Astronomy. Edited by Alan F. M. Moorwood and Iye Masanori. Proceedings of the SPIE, Volume 5492, pp. 410-420 (2004)., ed. A. F. M. Moorwood \& M. Iye, $410-420$

Struble, M. F., \& Rood, H. J. 1999, ApJS, 125, 35 
Tittley, E. R., \& Henriksen, M. 2005, ApJ, 618, 227

Tonry, J., \& Davis, M. 1979, AJ, 84, 1511

van der Marel, R. P., \& Franx, M. 1993, ApJ, 407, 525

Vikhlinin, A., Markevitch, M., \& Murray, S. S. 2001, ApJ, 551, 160

West, M. J., \& Bothun, G. D. 1990, ApJ, 350, 36
Yagi, M., Kashikawa, N., Sekiguchi, M., Doi, M., Yasuda, N., Shimasaku, K., \& Okamura, S. 2002, AJ, 123, 87

Zabludoff, A. I., Franx, M., \& Geller, M. J. 1993, ApJ, 419, 47 